ISSN 0103-9954

\title{
AVALIAÇÃO DE MODELOS POLINOMIAIS NÃO-SEGMENTADOS NA ESTIMATIVA DE DIÂMETROS E VOLUMES COMERCIAIS DE Pinus taeda
}

\author{
EVALUATION OF NON-SEGMENTED POLYNOMIAL TAPER MODELS FOR ESTIMATING \\ COMMERTIAL DIAMETERS AND VOLUMES OF Pinus taeda.
}

\author{
Adriana Leandra de Assis ${ }^{1}$ José Roberto Soares Scolforo ${ }^{2}$ \\ José Márcio de Mello ${ }^{3}$ Antônio Donizette de Oliveira ${ }^{4}$
}

\begin{abstract}
RESUMO
Este estudo foi realizado com os objetivos de: construir equações com potências de grandezas fracionárias, unitárias e de dezenas, na tentativa de representar melhor a ponta, a porção média e a base da árvore respectivamente; construir equações polinomiais compatíveis com uma equação volumétrica; avaliar a acuracidade de três modelos polinomiais não-segmentados para estimar diâmetros e volumes ao longo do fuste de Pinus taeda; verificar se o melhor modelo para estimar diâmetros ao longo do fuste é também o melhor para estimar volumes totais e parciais. A base de dados utilizada foi composta por 58 árvores de Pinus taeda cubadas rigorosamente pelo método de Smalian, nas propriedades da empresa PISA - Papel de Imprensa S/A, na região de Jaguariaíva - PR. As 58 árvores foram divididas em oito classes diamétricas, e procedeu-se ao ajuste de três modelos polinomiais não-segmentados para cada classe diamétrica e para o conjunto total dos dados. Os modelos não-segmentados testados foram o de Schöepfer (1966); o de Hradetzky (1976); e o de Goulding e Murray (1976). A acuracidade dos modelos para estimar di âmetros e volumes ao longo do fuste foi avaliada pr meio das seguintes estatísticas: coeficiente de determinação, erropadrão residual, análise gráfica dos perfis médios dos fustes, desvio médio em cada posição de medição ao longo do fuste, desvio-padrão das diferenças, soma de quadrados do resíduo relativo e resíduo percentual. Com base nas quatro últimas estatísticas, foi elaborado um "ranking" para detectar o modelo que propiciou estimativas mais acuradas de diâmetros e volumes em cada posição de medição do fuste, por ocasião da cubagem rigorosa. Como principais resultados, pode-se destacar que os modelos de Hradetzky (1976) é o mais estável na estimativa das duas variáveis estudadas. O modelo de Goulding e Murray (1976) é preciso para estimar diâmetros e volumes ao longo do fuste de Pinus taeda na região de estudo, apenas se o ajuste for por classe diamétrica. O modelo de Schöepfer (1966) mostrou estimativas tendenciosas ao longo de todo o fuste, em todas as classes diamétricas estudadas.
\end{abstract}

Palavras-chave: polinômio não-segmentado, função de afilamento.

\begin{abstract}
This study aimed at constructing equations with fractionary, unitary and ten magnitude power, in an attempt of representing the top, medium and basis portion of the stem; at constructing polynomial equations compatible with volume equations; at evaluating the accuracy of three non-segmented polynomial taper models for estimating diameters and volumes along the stem of Pinus taeda; and at verifying if the more accurated model for diameters estimates is also the more accurated for estimating partial and total volumes. The database used was composed of 58 trees of Pinus taeda cubed by the Smalian's method, in the farms of "PISA - Papel de Imprensa S/A" enterprise, located in Jaguariaíva county (PR). The 58 trees were separated in 8 diameter classes. The models were fitted for each diameter class and for the total group of data. Three non-segmented polynomial models were tested: Schöepfer (1966); Hradetzky (1976); and Goulding and Murray (1976). The accuracy of the models in estimating diameters and volumes along the stem of Pinus taeda was evaluated through the following statistics: coefficient of determination; residual standard error;

1. Engenheira Florestal, Msc., Acadêmica do Curso de Doutorado em Engenharia Florestal, Departamento de Ciências Florestais, Universidade Federal de Lavras, Caixa Postal 37, CEP 37200-000, Lavras, (MG).

2. Engenheiro Florestal, Dr., Professor Titular do Departamento de Ciências Florestais, Universidade Federal de Lavras, Caixa Postal 37, CEP 37200-000, Lavras, (MG).

3. Engenheiro Florestal, Msc., Professor Assistente do Departamento de Ciências Florestais, Universidade Federal de Lavras, Caixa Postal 37, CEP 37200-000, Lavras, (MG).

4. Engenheiro Florestal, Dr., Professor Adjunto do Departamento de Ciências Florestais, Universidade Federal de Lavras, Caixa Postal 37, CEP 37200-000, Lavras, (MG).
\end{abstract}


graphic analysis of the medium profile of the stem; mean deviation of the diameters and volumes for each position along the stem; standard deviation of the differences of the diameters and volumes; sum of square of the relative residue; and percentage of the residues of the diameters and volumes. Starting from the last four statistics, it was elaborated a ranking to detect the more accurated model for each position along the stem. It can be pointed out that among the non-segmented models tested, the model of Hradetzky (1976) is more stable than the others in the estimates of the two studied variables. Goulding and Murray (1976) model presented accurated estimates of diameters and volumes along the whole stem profile only when fitted by diameter classes. The model proposed by Schöepfer (1965) showed biased estimates along the whole stem profile, in all diameter classes.

Key words: non-segmented polynomial model, taper models.

\section{INTRODUÇÃO}

A necessidade de uma otimização do uso dos recursos florestais, dentro do conceito de múltiplos produtos da madeira, tem justificado e estimulado a realização de importantes estudos sobre o assunto. A aplicabilidade desses estudos é bastante clara, principalmente sob o ponto de vista do planejamento da produção de grandes empresas do setor florestal em que é fundamental garantir o fluxo contínuo de matéria-prima e a viabilidade econômica da atividade. Quando bem-manejadas e direcionadas para obter múltiplos produtos advindos da madeira, as florestas de produção garantem a maximização dos lucros na medida em que se adotam práticas com o objetivo de agregar valor à madeira. Além disso, a obtenção de múltiplos produtos reduz o impacto das oscilações do mercado sobre cada produto individualmente.

Mas, a obtenção dos múltiplos produtos advindos da madeira das florestas de produção passa necessariamente pelo conhecimento das características do ritmo de crescimento e da forma de seus indivíduos, bem como dos fatores que afetam essas duas variáveis. Por isso, o desenvolvimento de modelos matemáticos capazes de representar com acuracidade os perfis dos fustes têm-se tornado uma ferramenta cada vez mais importante dentro dos empreendimentos de base florestal. Em conseqüência da sua flexibilidade e ao amplo leque de informações que proporcionam sem aumento nos custos de inventário, as funções de afilamento têm ganhado espaço no meio florestal.

Dentre as diversas técnicas estatísticas de modelagem, destacam-se os modelos polinomiais não-segmentados que conseguem aliar eficiência a uma certa simplicidade de aplicação, quando comparados com os polinômios segmentados. Tais modelos têm sido estudados e algumas vezes apresentam deficiências na representação da base dos fustes o que pode comprometer as estimativas volumétricas. Por isso, de acordo com Rios (1997), Fischer (1997) e Rosot (1989) mostram o trabalho minucioso do pesquisador Hradetzky (1976), estudando as potências mais apropriadas na descrição da forma da árvore e apontando como sugestão a construção do modelo pelo método "stepwise", para expoentes variando de 0,005 a 25 . Assim, uma boa representação do tronco por meio de polinômios exige uma combinação apropriada de potências e que estas sejam submetidas ao processo de sele ção da regressão "passo a passo" (stepwise).

Há que se considerar ainda, as possibilidades de modifica ções desses modelos, objetivando suprir suas deficiências. Ainda existem poucos estudos sobre o assunto, tendo em vista que o grande impulso na aplicação dessas funções foi por causa da popularização das tecnologias na área de informática. Goulding e Murray (1976), trabalhando com Pinus radiata, propuseram uma modificação na função de forma de Kozak, Munro e Smith (1969), para torná-la compatível com uma equação de volume. No entanto, quando a equação foi ajustada aos dados, sua forma básica ficou deficiente na região do topo da árvore, não estimando diâmetro zero na ponta da árvore e, freqüentemente, fornecendo valores negativos $\mathrm{de}^{2}$ para várias combinações de DAP e $\mathrm{H}$. O ajuste da equação foi melhor quando esta foi ajustada com todos os expoentes do Polinômio do Quinto Grau, embora alguns termos não fossem significativos ao nível de 95\%; e sugeriram que, talvez, a inclusão de termos de ordem mais alta pudesse melhorar o desempenho do modelo.

Rosot (1989) utilizou o processo "stepwise" para construir modelos por classe de diâmetro. Concordando com Hradetzky (1976), concluiu que os modelos construídos pelo processo "stepwise" foram sempre mais precisos que o polinômio do quinto grau. Rosot (1989) observou ainda uma grande variação dos modelos nas diferentes classes diamétricas sugerindo assim, o ajuste por classe de di âmetro. 
Leite (1994), desenvolvendo um sistema de otimização para conversão de troncos e recuperação de multiprodutos da madeira baseado em Programação Dinâmica, considera a importância da modelagem da forma dos fustes dentro do sistema. Em seu estudo, emprega o modelo de Kozak, Mun ro e Smith (1969) e o modelo de Demaerschalk (1976) para analisar o "taper" dos troncos.

Importantes estudos envolvendo modelos polinomiais não-segmentados têm sido desenvolvidos no Brasil, destacando-se os trabalhos de Ahrens e Holbert (1981); Borges (1982), Silva (1982); Lima (1986), McTague, Batista e Steiner (1989); Kirchner, Figueiredo Filho, Scolforo et al. (1989); Leite, Guimarães e Campos (1995); Fischer (1997), Rios (1997), Scolforo, Rios, Oliveira et al. (1998), Assis (1998), Ferreira (1999) e Assis (2000). No entanto, dentre os estudos existentes, poucos abordam as estimativas volumétricas, quando, na verdade, um modelo que estime diâmetros satisfatoriamente não fornece necessariamente estimativas satisfat órias de volumes.

Assim, o presente estudo foi desenvolvido com os objetivos de:

a) Construir uma equação com potências de grandezas fracionárias, unitárias, e de dezenas, na tentativa de melhor representar a ponta, a porção intermediária e a base da árvore, respectivamente.

b) Construir uma equação polinomial compatível com uma equação volumétrica.

c) Avaliar a acuracidade de três modelos polinomiais não-segmentados para estimar diâmetros ao longo do fuste de Pinus taeda.

d) Avaliar a precisão dos modelos não-segmentados na estimativa de volumes totais e parciais de Pinus taeda.

e) Verificar se o melhor modelo para a estimativa dos diâmetros comerciais é também o melhor modelo para estimar volumes totais e comerciais de Pinus taeda na região de Jaguariaíva, PR.

\section{MATERIAL E MÉTODOS}

\section{Área de Estudo}

Os dados de Pinus taeda utilizados para este estudo são provenientes das propriedades da empresa PISA (Papel de Imprensa S/A), localizada no município de Jaguariaíva, estado do Paraná, entre os paralelos $24^{\circ}$ e $24^{\circ} 30^{\prime}$ de latitude sul e os meridianos $49^{\circ} 30^{\prime}$ e $50^{\circ}$ de longitude oeste de Greenwich, com altitude variando entre 700 e $1100 \mathrm{~m}$.

De acordo com a classificação de Koeppen, o clima da região é do tipo $\mathrm{Cfb}$, ou seja, subtropical quente-temperado, caracterizado por apresentar temperatura média inferior a $22^{\circ} \mathrm{C}$ no mês mais quente do ano. O mês mais chuvoso é janeiro cuja precipitação média é de $225,4 \mathrm{~mm}$ e não há estação seca definida (Golfari, Caser e Moura, 1978; Instituto Agronômico do Paraná, 1994).

Foram cubadas rigorosamente 58 árvores de Pinus taeda, com idade variando de 16 a 21 anos, e distribuídas em oito classes diamétricas, sendo que o menor valor das classes foi $15 \mathrm{~cm}$. A cubagem foi relativa, tomando-se medidas de diâmetro a $0 \%, 1 \%, 2 \%, 3 \%, 4 \%, 5 \%, 10 \%, 15 \%, 25 \%, 35 \%, 45 \%, 55 \%$, $65 \%, 75 \%, 85 \%$ e $95 \%$ da altura total da árvore, partindo de sua base. O cálculo dos volumes das seções foi feito com uso da fórmula de Smalian.

\section{Modelos Polinomiais Não-Segmentados}

O ajuste dos modelos polinomiais não-segmentados foi feito para cada uma das oito classes diamétricas e também desconsiderando o controle das classes diamétricas.

Os três modelos testados são apresentados, a seguir, nas suas formas de ajuste (obten ção de diâmetros ao longo do fuste) e nas suas formas que permitem estimar os volumes.

\section{Polinômio do Quinto Grau - Schöepfer (1966)}

O Polinômio do Quinto Grau tem a forma: 


$$
\frac{\mathrm{d}_{\mathrm{i}}}{\mathrm{D}}=\beta_{0}+\beta_{1}\left(\frac{\mathrm{h}_{\mathrm{i}}}{\mathrm{H}}\right)+\beta_{2}\left(\frac{\mathrm{h}_{\mathrm{i}}}{\mathrm{H}}\right)^{2}+\beta_{3}\left(\frac{\mathrm{h}_{\mathrm{i}}}{\mathrm{H}}\right)^{3}+\beta_{4}\left(\frac{\mathrm{h}_{\mathrm{i}}}{\mathrm{H}}\right)^{4}+\beta_{5}\left(\frac{\mathrm{h}_{\mathrm{i}}}{\mathrm{H}}\right)^{5}+\mathrm{e}_{\mathrm{i}}
$$

Sendo: $\beta_{\text {is }}=$ parâmetros a serem estimados; $d_{i}=$ diâmetro correspondente a qualquer altura $h_{i}$, especificada ou comercial $(\mathrm{cm}) ; \mathrm{D}=$ diâmetro a $1,3 \mathrm{~m}$ de altura $(\mathrm{cm}) ; \mathrm{H}=$ altura total $(\mathrm{m}) ; \mathrm{h}_{\mathrm{i}}=$ altura comercial (m); e $\mathrm{e}_{\mathrm{i}}=$ erro de estimativa.

Isolando $\mathrm{d}_{\mathrm{i}}$ obtém-se a função de afilamento por meio da qual pode-se estimar o diâmetro correspondente a qualquer altura na árvore, desde que fornecido o seu diâmetro a 1,3m de altura (DAP ou D) e a altura total.

$$
\mathrm{d}_{\mathrm{i}}=\mathrm{D}\left[\beta_{0}+\beta_{1}\left(\frac{\mathrm{h}_{\mathrm{i}}}{\mathrm{H}}\right)+\beta_{2}\left(\frac{\mathrm{h}_{\mathrm{i}}}{\mathrm{H}}\right)^{2}+\beta_{3}\left(\frac{\mathrm{h}_{\mathrm{i}}}{\mathrm{H}}\right)^{3}+\beta_{4}\left(\frac{\mathrm{h}_{\mathrm{i}}}{\mathrm{H}}\right)^{4}+\beta_{5}\left(\frac{\mathrm{h}_{\mathrm{i}}}{\mathrm{H}}\right)^{5}\right]+\mathrm{e}_{\mathrm{i}}
$$

Para integrar a função e obter a expressão que permite a estimativa dos volumes, fez-se a seguinte simplificação: $c_{0}=\beta_{0} ; \quad c_{1}=\frac{\beta_{1}}{\mathrm{H}} ; \quad c_{2}=\frac{\beta_{2}}{\mathrm{H}^{2}} ; \ldots \ldots . . ; c_{5}=\frac{\beta_{5}}{\mathrm{H}^{5}}$

Feita a simplificação, a expressão a ser integrada assume a forma:

$$
\mathrm{d}_{\mathrm{i}}=\mathrm{D}\left[\mathrm{c}_{0}+\mathrm{c}_{1} \cdot \mathrm{h}+\mathrm{c}_{2} \cdot \mathrm{h}^{2}+\mathrm{c}_{3} \cdot \mathrm{h}^{3}+\mathrm{c}_{4} \cdot \mathrm{h}^{4}+\mathrm{c}_{5} \cdot \mathrm{h}^{5}\right]+\mathrm{e}_{\mathrm{i}}
$$

$\mathrm{O}$ volume $(\mathrm{V})$ de um sólido de revolução é obtido pela integração de suas áreas seccionais $\left(\mathrm{g}_{\mathrm{i}}\right)$ entre o limite inferior $\left(\mathrm{h}_{1}\right)$ e o superior $\left(\mathrm{h}_{2}\right)$ que se deseja estabelecer. No caso de uma árvore, se o volume total é desejado, então $h_{1}=0$ e $h_{2}=$ altura total da árvore. A representação da integral é:

$$
\mathrm{V}=\int_{\mathrm{h}_{1}}^{\mathrm{h}_{2}} \mathrm{~g}_{\mathrm{i}} \delta \mathrm{h} \rightarrow \mathrm{V}=\int_{\mathrm{h}_{1}}^{\mathrm{h}_{2}} \frac{\pi * \mathrm{~d}_{\mathrm{i}}^{2}}{40000} \delta \mathrm{h} \rightarrow \mathrm{V}=\mathrm{K} \int_{\mathrm{h}_{1}}^{\mathrm{h}_{2}} \mathrm{~d}_{\mathrm{i}}^{2} \delta \mathrm{h}
$$

Sendo: $K=\frac{\pi}{40000}$

Então, substituindo (2) em (3), tem-se:

$$
\mathrm{V}=\mathrm{K} \cdot \mathrm{D}^{2} \cdot \int_{\mathrm{h}_{1}}^{\mathrm{h}_{2}}\left(\mathrm{c}_{0}+\mathrm{c}_{1} \mathrm{~h}_{\mathrm{i}}+\mathrm{c}_{2} \mathrm{~h}_{\mathrm{i}}^{2}+\mathrm{c}_{3} \mathrm{~h}_{\mathrm{i}}^{3}+\mathrm{c}_{4} \mathrm{~h}_{\mathrm{i}}^{4}+\mathrm{c}_{5} \mathrm{~h}_{\mathrm{i}}^{5}\right)^{2} \delta \mathrm{h}_{\mathrm{i}}+\mathrm{e}_{\mathrm{i}}
$$

Integrando (4), obtém-se a expressão que propicia obter os sortimentos ou volumes comerciais correspondentes a qualquer porção da árvore, além do volume total, se este for desejado.

$$
\begin{aligned}
& \mathrm{V}=\mathrm{K} \cdot \mathrm{D}^{2} \cdot\left[\mathrm{c}_{0}^{2} \mathrm{~h}_{\mathrm{i}}+\mathrm{c}_{0} \mathrm{c}_{1} \mathrm{~h}_{\mathrm{i}}^{2}+\left(2 / 3 \mathrm{c}_{0} \mathrm{c}_{2}+1 / 3 \mathrm{c}_{1}^{2}\right) \mathrm{h}_{\mathrm{i}}^{3}+\left(1 / 2 \mathrm{c}_{0} \mathrm{c}_{3}+1 / 2 \mathrm{c}_{1} \mathrm{c}_{2}\right) \mathrm{h}_{\mathrm{i}}^{4}+\left(2 / 5 \mathrm{c}_{0} \mathrm{c}_{4}+\right.\right. \\
& \left.+2 / 5 \mathrm{c}_{1} \mathrm{c}_{3}+1 / 5 \mathrm{c}_{2}^{2}\right) \mathrm{h}_{\mathrm{i}}^{5}+\left(1 / 3 \mathrm{c}_{0} \mathrm{c}_{5}+1 / 3 \mathrm{c}_{1} \mathrm{c}_{4}+1 / 3 \mathrm{c}_{2} \mathrm{c}_{3}\right) \mathrm{h}_{\mathrm{i}}^{6}+\left(2 / 7 \mathrm{c}_{1} \mathrm{c}_{5}+2 / 7 \mathrm{c}_{2} \mathrm{c}_{4}+1 / 7 \mathrm{c}_{3}^{2}\right) \mathrm{h}_{\mathrm{i}}^{7}+ \\
& \left.+\left(1 / 4 \mathrm{c}_{2} \mathrm{c}_{5}+1 / 4 \mathrm{c}_{3} \mathrm{c}_{4}\right) \mathrm{h}_{\mathrm{i}}^{8}+\left(2 / 9 \mathrm{c}_{3} \mathrm{c}_{5}+1 / 9 \mathrm{c}_{4}^{2}\right) \mathrm{h}_{\mathrm{i}}^{9}+1 / 5 \mathrm{c}_{4} \mathrm{c}_{5} \mathrm{~h}_{\mathrm{i}}^{10}+1 / 1 \mathrm{c}_{5}^{2} \mathrm{~h}_{\mathrm{i}}^{11}\right]_{\mathrm{h}_{1}}^{\mathrm{h}_{2}}+\mathrm{e}_{\mathrm{i}}
\end{aligned}
$$

\section{Polinômio de Potências Fracionárias e Inteiras - Hradetzky (1976)}

Esse modelo se diferencia do polinômio do quinto grau por apresentar um mix de potências inteiras e fracionárias como tentativa de melhor representar o perfil da árvore. Hradetzky (1976) sugeriu a utilização de potências inteiras da ordem de dezenas para representar a base de árvore, em conjunto com potências fracionárias para representar a porção superior do fuste. Esses modelos foram construídos por classe diamétrica e para o conjunto total dos dados, utilizando o procedimento "Stepwise". Nos trabalhos de Fischer (1997), Rios (1997), Assis (1998), e Ferreira (1999), os expoentes utilizados no processo de construção dos modelos, por meio do procedimento "stepwise", variaram de 0,005 a 25 . No entanto, 
observou-se que estes dois extremos eram freqüentemente selecionados para formar o modelo o que poderia sugerir a inclusão de potências maiores e menores no processo de sele ção de variáveis. Por esse motivo, este estudo incluiu uma série de outros expoentes, além dos testados pelos autores supracitados. Os expoentes testados foram: 0,$00001 ; 0,00005 ; 0,0009 ; 0,0007 ; 0,0006 ; 0,0004 ; 0,0002 ; 0,0001 ; 0,009 ; 0,008 ; 0,007$; 0,$006 ; 0,005 ; 0,004 ; 0,09 ; 0,08 ; 0,07 ; 0,06 ; 0,05 ; 0,04 ; 0,03 ; 0,02 ; 0,01 ; 0,9 ; 0,8 ; 0,7 ; 0,6 ; 0,5 ; 0,4 ; 0,3 ; 0,2$; 0,$1 ; 1 ; 2 ; 3 ; 4 ; 5 ; 10 ; 15 ; 20 ; 25 ; 30 ; 35 ; 40 ; 45 ; 50 ; 55 ; 60 ; 65 ; 70 ; 75 ; 80 ; 85 ; 90$ e 95.

A forma de ajuste do polinômio é:

$$
\mathrm{d}_{\mathrm{i}}=\mathrm{D}\left[\beta_{0}+\beta_{1}\left(\frac{\mathrm{h}_{\mathrm{i}}}{\mathrm{H}}\right)^{\mathrm{p} 1}+\beta_{2}\left(\frac{\mathrm{h}_{\mathrm{i}}}{\mathrm{H}}\right)^{\mathrm{p} 2}+\ldots+\beta_{\mathrm{n}}\left(\frac{\mathrm{h}_{\mathrm{i}}}{\mathrm{H}}\right)^{\mathrm{pn}}\right]+\mathrm{e}_{\mathrm{i}}
$$

Em que: $\mathrm{d}_{\mathrm{i}}, \mathrm{D}, \mathrm{h}_{\mathrm{i}}, \mathrm{H}, \beta_{\mathrm{i}}, \mathrm{e} \mathrm{e}_{\mathrm{i}}$ foram definidos anteriormente.

Ao simplificar a expressão por: $c_{0}=\beta_{0}$ e $\quad c_{i}=\left(\beta_{\mathrm{i}} / \mathrm{H}^{\mathrm{p}_{\mathrm{j}}}\right)$, em que $\mathrm{i}=1,2, \ldots, \mathrm{N}$; e $\mathrm{p}_{\mathrm{j}}=$ expoentes selecionados por meio do processo "stepwise", a express ão (6) assume a forma:

$$
\mathrm{d}_{\mathrm{i}}=\mathrm{D}\left(\mathrm{c}_{0}+\mathrm{c}_{1} \mathrm{~h}_{1}^{\mathrm{p}_{1}}+\mathrm{c}_{2} \mathrm{~h}_{2}^{\mathrm{p}_{2}}+\ldots+\mathrm{c}_{\mathrm{n}} \mathrm{h}_{\mathrm{n}}^{\mathrm{p}_{\mathrm{n}}}\right)+\mathrm{e}_{\mathrm{i}}
$$

O volume total ou de qualquer porção da árvore (sortimento) é obtido pela resolução da integral do polinômio (7), após sua substituição na expressão (5). O resultado desta é:

$$
\begin{aligned}
& V=K \cdot D^{2} \cdot\left[c_{0}^{2} h_{i}+2 c_{0} c_{1}\left(\frac{h_{i}^{\left(p_{1}+1\right)}}{p_{1}+1}\right)+2 c_{0} c_{2}\left(\frac{h_{i}^{\left(p_{2}+1\right)}}{p_{2}+1}\right)+\ldots+2 c_{0} c_{(n-1)}\left(\frac{h_{i}^{\left(p_{(n-1)}+1\right)}}{p_{(n-1)}+1}\right)+2 c_{0} c_{n}\left(\frac{h_{i}^{\left(p_{n}+1\right)}}{p_{n}+1}\right)+2 c_{1} c_{2}\left(\frac{h_{i}{ }_{i}^{\left(p_{1}+p_{2}+1\right)}}{p_{1}+p_{2}+1}\right)+\right. \\
& +\ldots+c_{1} c_{(n-1)}\left(\frac{h_{i}{ }^{\left(p_{1}+p_{(n-1)}+1\right)}}{p_{1}+p_{(n-1)}+1}\right)+2 c_{1} c_{n}\left(\frac{h_{i}{ }^{\left(p_{1}+p_{n}+1\right)}}{p_{1}+p_{n}+1}\right)+2 c_{2} c_{(n-1)}\left(\frac{h_{i}{ }^{\left(p_{2}+p_{(n-1)}+1\right)}}{p_{2}+p_{(n-1)}+1}\right)+2 c_{2} c_{n}\left(\frac{h_{i}{ }^{\left(p_{2}+p_{n}+1\right)}}{p_{2}+p_{n}+1}\right)+ \\
& \left.+\ldots+2 c_{(n-1)} c_{n}\left(\frac{h_{i}^{\left(p_{(n-1)}+p_{n}+1\right)}}{p_{(n-1)}+p_{n}+1}\right)+c_{1}^{2}\left(\frac{h_{i}^{\left(2 p_{1}+1\right)}}{2 p_{1}+1}\right)+c_{2}^{2}\left(\frac{h_{i}^{\left(2 p_{2}+1\right)}}{2 p_{2}+1}\right)+\ldots+c_{(n-1)}^{2}\left(\frac{h_{i}^{\left(2 p_{(n-1)}+1\right)}}{2 p_{(n-1)}}\right)+c_{n}^{2}\left(\frac{h_{i}^{\left(2 p_{n}+1\right)}}{2 p_{n}+1}\right)\right]_{h_{1}}^{h_{2}}+e_{i}
\end{aligned}
$$

\section{Modelo de Goulding e Murray (1976)}

Goulding e Murray (1976) propuseram uma função de afilamento polinomial compatível com uma equação de volume. Trata-se na verdade de uma modificação do Polinômio do Quinto Grau, conforme apresentado a seguir.

A forma geral do polinômio compatível de Goulding e Murray (1976) é:

$$
\mathrm{d}_{\mathrm{i}}^{2}=\frac{\hat{\mathrm{V}}}{\mathrm{KH}}\left[\beta_{1}\left(\frac{\mathrm{L}}{\mathrm{H}}\right)+\beta_{2}\left(\frac{\mathrm{L}}{\mathrm{H}}\right)^{2}+\beta_{3}\left(\frac{\mathrm{L}}{\mathrm{H}}\right)^{3}+\ldots+\beta_{\mathrm{n}}\left(\frac{\mathrm{L}}{\mathrm{H}}\right)^{\mathrm{n}}\right]+\mathrm{e}_{\mathrm{i}}
$$

Sendo: $\beta_{i}=$ parâmetros a serem estimados para os quais $\sum_{i=1}^{n} \frac{\beta_{i}}{(i+1)}=1 ; d_{i}=$ diâmetro comercial $(\mathrm{cm}) ; \mathrm{K}=\pi / 40000 ; \hat{\mathrm{V}}=$ volume estimado pela equação de volume individual $\left(\mathrm{m}^{3}\right) ; \mathrm{H}=$ altura total $(\mathrm{m}) ; \mathrm{h}_{\mathrm{i}}$ $=$ altura comercial $(\mathrm{m}) ; \mathrm{L}=(\mathrm{H}-\mathrm{h}) ; \mathrm{e}_{\mathrm{i}}=$ erro de estimativa.

O modelo volumétrico utilizado para estimar os volumes totais foi: $V=\beta_{1} D^{2} H+\beta_{2} H+e_{i}$ sendo $\mathrm{D}=$ diâmetro a $1,3 \mathrm{~m}$ do solo; $\mathrm{V}=$ volume real da árvore; $\beta_{\mathrm{i}}, \mathrm{H}$ e $\mathrm{e}_{\mathrm{i}}$ definidos anteriormente.

A forma linear do polinômio proposto (8), que permite a seleção das variáveis pela técnica "stepwise" é, a seguinte:

$$
\frac{\mathrm{d}^{2} \mathrm{KH}}{\mathrm{V}}-\frac{2 \mathrm{~L}}{\mathrm{H}}=\beta_{2}^{\prime}\left(3\left(\frac{\mathrm{L}}{\mathrm{H}}\right)^{2}-\frac{2 \mathrm{~L}}{\mathrm{H}}\right)+\beta_{3}^{\prime}\left(4\left(\frac{\mathrm{L}}{\mathrm{H}}\right)^{3}-\frac{2 \mathrm{~L}}{\mathrm{H}}\right)+\beta_{\mathrm{n}}^{\prime}\left((\mathrm{n}+1)\left(\frac{\mathrm{L}}{\mathrm{H}}\right)^{\mathrm{n}}-\frac{2 \mathrm{~L}}{\mathrm{H}}\right)+\mathrm{e}_{\mathrm{i}}
$$




$$
\begin{aligned}
& \text { Após o ajuste de (9), os coeficientes do modelo (8) podem ser calculados como: } \beta_{1}=2 \cdot\left(1-\sum_{\mathrm{i}=2}^{\mathrm{n}} \beta_{\mathrm{i}}^{\prime}\right) \\
& \beta_{2}=3 \cdot \beta_{2}^{\prime} ; \beta_{\mathrm{n}}=(\mathrm{n}+1) \cdot \beta_{\mathrm{n}}^{\prime} .
\end{aligned}
$$

Integrando-se a expressão (8), obtém-se a fórmula para cálculo dos volumes comerciais. É importante observar que, como a expressão utiliza a distância do topo da árvore até um ponto h qualquer, os volumes estimados correspondem aos volumes da ponta da árvore até uma altura h, e não ao volume da base da árvore até uma altura comercial.

Assim, o volume total ou o dos sortimentos pode ser obtido como:

$$
V c=\frac{V}{H}\left[\frac{\beta_{1} L^{2}}{2 H}+\frac{\beta_{2} L^{3}}{3 H^{2}}+\frac{\beta_{3} L^{4}}{4 H^{3}}+\ldots+\frac{\beta_{n} L^{(n+1)}}{(n+1) H^{n}}\right]_{0}^{h}+e_{i}
$$

Sendo $\mathrm{Vc}=$ volume da ponta da árvore até a altura $\mathrm{h}\left(\mathrm{em} \mathrm{m}^{3}\right) ; \beta_{\mathrm{i}}, \mathrm{L}, \mathrm{H} \mathrm{e} \mathrm{V}$, definidos anteriormente.

Os expoentes testados foram $2,3,4,5,6,7,8,9,10,11,12,13,14,15,16,17,18,19,20,21,22$, $23,24,25,26,27,28,29,30,31,32,33,34,35,36$ e 37 .

Em alguns casos, a equação construída pelo processo "stepwise" apresenta uma certa deficiência para estimar os diâmetros da porção superior dos fustes. Por isso, para cada classe diamétrica e para o conjunto total dos dados, esse modelo foi ajustado de duas maneiras: um ajuste "stepwise" sem forçar a entrada de variáveis e um outro ajuste forçando a entrada de quatro variáveis (expoentes 2, 3, 4 e 5), transformando o modelo num Polinômio do Quinto Grau com adição de variáveis pelo processo "stepwise". Nos casos em que a inclusão de quatro variáveis forçadas no modelo ocasionou deficiência nas estimativas dos diâmetros na ponta das árvores, foi feito um terceiro ajuste, forçando a entrada de três variáveis. A adoção desse procedimento propiciou o desenvolvimento de duas equa ções para essa alternativa de ajuste.

\section{Acuracidade dos modelos de afilamento}

O modelo do Polinômio do Quinto Grau, o de Potências Fracionárias e Inteiras, o modelo polinomial compatível com o modelo volumétrico, e este último com entrada de variáveis forçadas tiveram sua acuracidade avaliada mediante várias estatísticas, como o coeficiente de determinação $\left(\mathrm{R}^{2}\right)$, e o erro-padrão da estimativa (Syx). Estas foram utilizadas apenas para verificar se os modelos apresentaram ajustes satisfatórios de uma maneira geral. Adicionalmente, foram calculadas estatísticas como aquelas utilizadas por Parresol, Hotvedt e Cao (1987) e Figueiredo Filho, Borders e Hitch (1996). Tais estat ísticas permitem uma análise mais detalhada do desempenho das estimativas ao longo de todo o fuste, uma vez que foram calculadas para cada altura relativa em que foram tomados os diâmetros por ocasião da cubagem rigorosa. As variáveis avaliadas foram os diâmetros estimados em cada posição de medição (alturas relativas) e os volumes parciais correspondentes a essas mesmas posições, além do volume total. A Tabela 1 apresenta as estatísticas calculadas para verificar a acuracidade das estimativas de di âmetros e volumes.

Com base nessas estatísticas, foi elaborado um ranking para expressar, de forma sumarizada, o desempenho dos modelos de afilamento testados para estimar diâmetros ao longo do fuste, e os volumes totais e parciais das árvores em estudo. Esse ranking foi feito para cada classe diamétrica e também para o conjunto das árvores sem o controle das classes diamétricas, para as variáveis diâmetro e volume. Considerando cada uma das estatísticas da Tabela 1, foi atribuída nota 1 à equação que apresentou maior acuracidade em cada posição relativa de medição e, assim sucessivamente, até a nota 4 para a equação menos acurada. No caso de mesmo valor em qualquer das estatísticas para diferentes equações, estas receberam a mesma nota. 
TABELA 1: Estatísticas utilizadas para avaliação das estimativas dos diâmetros e volumes comerciais de Pinus taeda.

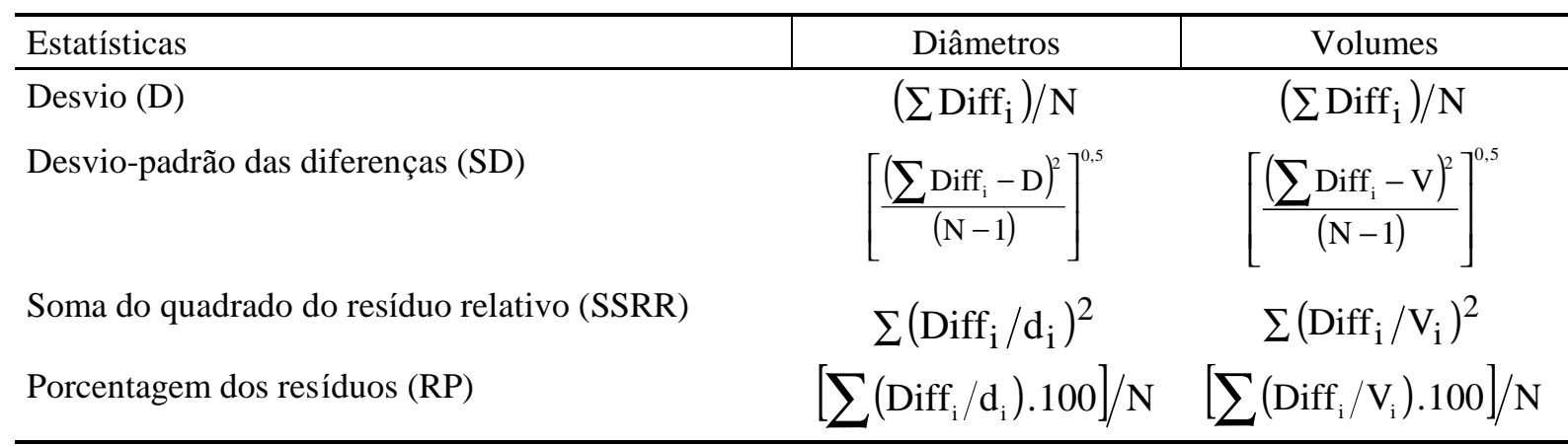

Em que: Diff $_{\mathrm{i}}=\left(V_{i}-\hat{V}_{i}\right)$ ou $\left(\mathrm{d}_{\mathrm{i}}-\hat{\mathrm{d}}_{\mathrm{i}}\right)$ é o desvio dos volumes ou dos diâmetros; $\mathrm{V}_{\mathrm{i}}=$ volume total ou comercial até $\wedge$

o diâmetro i da árvore; $\mathrm{V}_{\mathrm{i}}=$ volume total ou comercial estimado; $\mathrm{d}_{\mathrm{i}}=$ diâmetro da árvore na posição i do fuste; $\mathrm{d}_{\mathrm{i}}=$ diâmetro estimado da árvore para a posição i do fuste; $\mathrm{N}=$ número de árvores.

\section{RESULTADOS E DISCUSSÃO}

Ajuste dos modelos

Os parâmetros estimados para todos os modelos ajustados são apresentados na Tabela 2 com as respectivas medidas de precisão: coeficiente de determinação corrigido $\left(\mathrm{R}^{2}\right)$ e erro-padrão da estimativa corrigido e em percentagem (Syx\%); para todas as classes diamétricas e para o ajuste feito com todos os dados.

É importante observar que as estatísticas tradicionais, apresentadas na Tabela 2, não retratam o desempenho das funções de afilamento, considerando que são médias que não consideram a posição da estimativa ao longo dos fustes. Portanto, servem apenas como um indicativo da correlação existente entre as variáveis envolvidas nos modelos testados. Nesse contexto, todos os modelos apresentaram coeficientes de determinação $\left(\mathrm{R}^{2}\right)$ satisfatórios, sendo os valores mais baixos obtidos pelo modelo de Goulding e Murray (1976). Quanto ao erro-padrão da estimativa (Syx\%), os valores não ultrapassaram 8,85\%, estando, na sua maioria, situados entre $3 \%$ e $7 \%$.

TABELA 2: Parâmetros estimados para os modelos não-segmentados e respectivas medidas de precisão, para as oito classes diamétricas e para o conjunto total dos dados.

\begin{tabular}{|c|c|c|c|c|c|c|c|c|c|c|}
\hline \multicolumn{11}{|c|}{ Polinômio do quinto grau } \\
\hline \multirow{2}{*}{$\begin{array}{l}\text { Classe de } \\
\text { diâmetro }\end{array}$} & \multicolumn{10}{|c|}{ Parâmetros estimados } \\
\hline & $\hat{\beta}_{0}$ & $\hat{\beta}_{1}$ & \multicolumn{2}{|c|}{$\hat{\beta}_{2}$} & \multicolumn{2}{|r|}{$\hat{\beta}_{3}$} & $\hat{\beta}_{4}$ & $\hat{\beta}_{5}$ & $\mathrm{R}^{2} \%$ & Syx $\%$ \\
\hline $17,5 \mathrm{~cm}$ & 1,21133 & $-4,77338$ & 23,00 & 770 & -53 & 26663 & 54,85541 & $-21,04009$ & 99,53 & 6,08 \\
\hline $22,5 \mathrm{~cm}$ & 1,18248 & $-4,25753$ & 20,30 & 347 & & 57934 & 47,31224 & $-17,96276$ & 99,66 & 5,11 \\
\hline $27,5 \mathrm{~cm}$ & 1,22205 & $-5,19014$ & 25,40 & 085 & & 10947 & 56,64449 & $-20,98704$ & 99,56 & 5,75 \\
\hline $32,5 \mathrm{~cm}$ & 1,18811 & $-4,30375$ & 20,38 & 717 & & 63349 & 44,64738 & $-16,29322$ & 99,66 & 4,95 \\
\hline $37,5 \mathrm{~cm}$ & 1,17885 & $-4,29564$ & 20,04 & 547 & & 92816 & 41,87355 & $-14,88609$ & 99,68 & 4,66 \\
\hline $42,5 \mathrm{~cm}$ & 1,14462 & $-3,73697$ & 17,45 & 561 & & 87629 & 35,11167 & $-12,10736$ & 99,67 & 4,76 \\
\hline $47,5 \mathrm{~cm}$ & 1,15302 & $-4,1261 \mathrm{C}$ & 19,08 & 363 & & 50658 & 36,75295 & $-12,37027$ & 99,80 & 3,73 \\
\hline $52,5 \mathrm{~cm}$ & 1,15433 & $-3,98529$ & 18,03 & 352 & & 02445 & 34,00589 & $-11,19119$ & 99,82 & 3,59 \\
\hline TOTAL & 1,16118 & $-4,08534$ & 18,93 & 462 & & 06565 & 38,45649 & $-13,41035$ & 99,66 & 4,93 \\
\hline \multicolumn{11}{|c|}{ Polinômio de potências inteiras e fracionárias } \\
\hline \multirow{2}{*}{$\begin{array}{l}\text { Classe de } \\
\text { diâmetro }\end{array}$} & \multicolumn{10}{|c|}{ Parâmetros estimados } \\
\hline & $\hat{\beta}_{0}$ & $\hat{\beta}_{1}$ & $\hat{\beta}_{2}$ & & & $\hat{\beta}_{4}$ & $\hat{\beta}_{5}$ & $\hat{\beta}_{6}$ & $\mathrm{R}^{2} \%$ & Syx\% \\
\hline $17,5 \mathrm{~cm}$ & 1,3346 & 748,306 & $-748,823$ & $-0,3$ & 066 & $-0,2759$ & $-0,23452$ & & 98,29 & 6,04 \\
\hline Potência & & 0,00001 & 0,0001 & & & 3 & 10 & & & \\
\hline
\end{tabular}

Continua ... 
TABELA 2: Continuação ...

\begin{tabular}{|c|c|c|c|c|c|c|c|c|c|}
\hline \multicolumn{10}{|c|}{ Polinômio de potências inteiras e fracionárias } \\
\hline \multirow{2}{*}{$\begin{array}{l}\text { Classe de } \\
\text { diâmetro }\end{array}$} & \multicolumn{9}{|c|}{ Parâmetros estimados } \\
\hline & $\hat{\beta}_{0}$ & $\hat{\beta}_{1}$ & $\hat{\beta}_{2}$ & $\hat{\beta}_{3}$ & $\hat{\beta}_{4}$ & $\hat{\beta}_{5}$ & $\hat{\beta}_{6}$ & $\mathrm{R}^{2} \%$ & Syx\% \\
\hline $17,5 \mathrm{~cm}$ & 1,3346 & 748,306 & $-748,823$ & $-0,30066$ & $-0,27598$ & $-0,23452$ & & 98,29 & 6,04 \\
\hline Potência & & 0,00001 & 0,0001 & 1 & 3 & 10 & & & \\
\hline $22,5 \mathrm{~cm}$ & 1,2812 & 0,16188 & $-0,81103$ & $-0,61496$ & & & & 98,71 & 5,19 \\
\hline Potência & & 0,00001 & 0,2 & 4 & & & & & \\
\hline $27,5 \mathrm{~cm}$ & 1,3432 & 1031,02 & $-1031,64$ & $-0,61118$ & $-0,11271$ & & & 98,84 & 5,15 \\
\hline Potência & & 0,00001 & 0,0001 & 3 & 10 & & & & \\
\hline \multicolumn{10}{|c|}{ Polinômio de potências inteiras e fracionárias } \\
\hline \multirow{2}{*}{$\begin{array}{l}\text { Classe de } \\
\text { diâmetro }\end{array}$} & \multicolumn{9}{|c|}{ Parâmetros estimados } \\
\hline & $\hat{\beta}_{0}$ & $\hat{\beta}_{1}$ & $\hat{\beta}_{2}$ & $\hat{\beta}_{3}$ & $\hat{\beta}_{4}$ & $\hat{\beta}_{5}$ & $\hat{\beta}_{6}$ & $\mathrm{R}^{2} \%$ & Syx\% \\
\hline $32,5 \mathrm{~cm}$ & 1,2693 & 1,16649 & 6,77383 & $-2,10279$ & $-6,79914$ & $-0,30551$ & & 98,83 & 5,23 \\
\hline Potência & & 0,00001 & 0,9 & 0,1 & 1 & 5 & & & \\
\hline $37,5 \mathrm{~cm}$ & 1,2834 & 0,33697 & 2,74425 & $-1,37104$ & $-2,59193$ & $-0,57642$ & 0,17677 & 99,21 & 4,38 \\
\hline Potência & & 0,00001 & 0,8 & 0,2 & 1 & 5 & 10 & & \\
\hline $42,5 \mathrm{~cm}$ & 1,2302 & 17,6675 & $-18,1341$ & $-0,84111$ & 0,13999 & $-0,06244$ & & 98,84 & 5,13 \\
\hline Potência & & 0,00001 & 0,004 & 3 & 20 & 95 & & & \\
\hline $47,5 \mathrm{~cm}$ & 1,2162 & 134,119 & $-134,338$ & $-0,32867$ & $-1,55917$ & 1,53847 & $-0,64989$ & 99,30 & 4,09 \\
\hline Potência & & 0,00001 & 0,0004 & 0,2 & 5 & 10 & 25 & & \\
\hline $52,5 \mathrm{~cm}$ & 1,2149 & 0,20294 & $-0,78977$ & $-1,56782$ & 1,60299 & $-0,66376$ & & 99,42 & 3,93 \\
\hline Potência & & 0,00001 & 0,2 & 5 & 10 & 15 & & & \\
\hline TOTAL & 1,2606 & 0,26445 & $-1,04086$ & 0,66834 & $-1,02726$ & $-0,12574$ & & 98,63 & 5,66 \\
\hline Potência & & 0,00001 & 0,2 & 1 & 2 & 5 & & & \\
\hline \multicolumn{10}{|c|}{ Modelo polinomial compatível com modelo volumétrico } \\
\hline \multirow{2}{*}{$\begin{array}{l}\text { Classe de } \\
\text { diâmetro }\end{array}$} & \multicolumn{9}{|c|}{ Parâmetros estimados } \\
\hline & $\hat{\beta}_{2}$ & $\hat{\beta}_{3}$ & $\hat{\beta}_{4}$ & $\hat{\beta}_{5}$ & $\hat{\beta}_{6}$ & $\hat{\beta}_{7}$ & $\hat{\beta}_{8}$ & $\mathrm{R}^{2} \%$ & Syx \% \\
\hline $17,5 \mathrm{~cm}$ & 54,449 & $-1312,13$ & 5973,463 & $-7187,96$ & 2472,236 & & & 90,64 & 5,84 \\
\hline Potência & 28 & 33 & 35 & & 37 & & & & \\
\hline $22,5 \mathrm{~cm}$ & 26,079 & $-51,945$ & 25,91029 & & & & & 78,84 & 6,57 \\
\hline Potência & 35 & 36 & 37 & & & & & & \\
\hline $27,5 \mathrm{~cm}$ & $-83,827$ & 525,877 & $-717,104$ & 275,0822 & & & & 88,56 & 7,03 \\
\hline Potência & 33 & 35 & 36 & 37 & & & & & \\
\hline $32,5 \mathrm{~cm}$ & 2,6068 & $-2,4509$ & 0,693346 & $-0,85055$ & 0,850809 & & & 91,50 & 4,45 \\
\hline Potência & 02 & 03 & 05 & 36 & 37 & & & & \\
\hline $37,5 \mathrm{~cm}$ & 3,9157 & $-5,0550$ & 2,28663 & $-39,1088$ & 394,1856 & $-594,786$ & 239,6783 & 94,71 & 3,89 \\
\hline Potência & 02 & 03 & 04 & 32 & 35 & 36 & 37 & & \\
\hline $42,5 \mathrm{~cm}$ & 4,6509 & $-6,0745$ & 2,77967 & $-56,5435$ & 547,7153 & $-815,662$ & 324,4179 & 83,04 & 5,06 \\
\hline Potência & 02 & 03 & 04 & 32 & 35 & 36 & 37 & & \\
\hline $47,5 \mathrm{~cm}$ & 4,4014 & $-5,1467$ & 2,042907 & $-0,35525$ & 0,372594 & & & 84,86 & 5,04 \\
\hline Potência & 02 & 03 & 04 & 36 & 37 & & & & \\
\hline $52,5 \mathrm{~cm}$ & 3,7772 & $-3,8624$ & 1,35513 & 0,03135 & & & & 91,64 & 4,43 \\
\hline Potência & 02 & 03 & 04 & 37 & & & & & \\
\hline TOTAL & 3,8186 & $-4,9011$ & 2,19546 & $-39,0608$ & 389,4872 & $-585,549$ & 235,0927 & 87,16 & 5,46 \\
\hline Potência & 02 & 03 & 04 & 32 & 35 & 36 & 37 & & \\
\hline
\end{tabular}

Continua ... 
TABELA 2: Continuação ...

\begin{tabular}{|c|c|c|c|c|c|c|c|c|c|c|}
\hline \multicolumn{11}{|c|}{ Polinômio compatível com modelo volumétrico, com inclusão de variáveis } \\
\hline \multirow{2}{*}{$\begin{array}{c}\text { Classe de } \\
\text { diâmetro }\end{array}$} & \multicolumn{10}{|c|}{ Parâmetros estimados } \\
\hline & $\hat{\beta}_{2}$ & $\hat{\beta}_{3}$ & $\hat{\beta}_{4}$ & $\hat{\beta}_{5}$ & $\hat{\beta}_{6}$ & $\hat{\beta}_{7}$ & $\hat{\beta}_{8}$ & $\hat{\beta}_{9}$ & $\mathrm{R}^{2} \%$ & Syx $\%$ \\
\hline $17,5 \mathrm{~cm}$ & $-0,2029$ & 3,8770 & $-7,825$ & 4,5778 & $-55,061$ & 797,467 & $-1270,88$ & 528,23 & 90,57 & 5,71 \\
\hline Potência & 02 & 03 & 04 & 05 & 31 & 35 & 36 & 37 & & \\
\hline $22,5 \mathrm{~cm}$ & 7,052 & $-16,971$ & 18,259 & $-7,307$ & 51,604 & $-100,82$ & 49,360 & & 79,54 & 6,35 \\
\hline Potência & 02 & 03 & 04 & 05 & 35 & 36 & 37 & & & \\
\hline $27,5 \mathrm{~cm}$ & 7,639 & $-17,733$ & 18,582 & $-7,336$ & 53,895 & $-105,41$ & 51,666 & & 88,95 & 5,71 \\
\hline Potência & 02 & 03 & 04 & 05 & 35 & 36 & 37 & & & \\
\hline $32,5 \mathrm{~cm}$ & 2,7176 & $-2,7291$ & 0,2958 & 0,5800 & $-0,8401$ & 0,8409 & & & 91,50 & 4,46 \\
\hline Potência & 02 & 03 & 04 & 05 & 36 & 37 & & & & \\
\hline $47,5 \mathrm{~cm}$ & 2,6268 & $-0,6218$ & $-2,8364$ & 1,8915 & $-0,5439$ & 0,55057 & & & 84,92 & 4,81 \\
\hline Potência & 02 & 03 & 04 & 05 & 36 & 37 & & & & \\
\hline \multicolumn{11}{|c|}{ Polinômio compatível com modelo volumétrico, com inclusão de variáveis } \\
\hline \multirow{2}{*}{$\begin{array}{l}\text { Classe de } \\
\text { diâmetro }\end{array}$} & \multicolumn{10}{|c|}{ Parâmetros estimados } \\
\hline & $\hat{\beta}_{2}$ & $\hat{\beta}_{3}$ & $\hat{\beta}_{4}$ & $\hat{\beta}_{5}$ & $\hat{\beta}_{6}$ & $\hat{\beta}_{7}$ & $\hat{\beta}_{8}$ & $\hat{\beta}_{9}$ & $\mathrm{R}^{2} \%$ & Syx $\%$ \\
\hline $52,5 \mathrm{~cm}$ & 1,3787 & 2,7779 & $-6,3330$ & 3,1721 & $-0,5917$ & 0,5934 & & & 85,67 & 4,24 \\
\hline Potência & 02 & 03 & 04 & 05 & 36 & 37 & & & & \\
\hline \multirow{3}{*}{\multicolumn{2}{|c|}{$\begin{array}{c}\text { Classe } \\
\text { de } \\
\text { diâmetro }\end{array}$}} & \multicolumn{9}{|c|}{ Equação de volume individual para GOULDING \& MURRAY (1976) } \\
\hline & & \multicolumn{9}{|c|}{ Parâmetros e estatísticas estimados } \\
\hline & & \multicolumn{2}{|r|}{$\hat{\beta}_{1}$} & \multicolumn{2}{|r|}{$\hat{\beta}_{2}$} & \multicolumn{3}{|c|}{$\mathrm{R}^{2} \%$} & \multicolumn{2}{|c|}{ Syx \% } \\
\hline $17,5 \mathrm{~cm}$ & & \multicolumn{2}{|c|}{0,000034951} & \multirow{2}{*}{\multicolumn{2}{|c|}{$\begin{array}{r}0,001033 \\
-0,006067\end{array}$}} & \multicolumn{3}{|c|}{99,41} & \multicolumn{2}{|l|}{7,99} \\
\hline $22,5 \mathrm{~cm}$ & & \multicolumn{2}{|c|}{0,000049335} & & & \multicolumn{3}{|c|}{99,30} & \multicolumn{2}{|l|}{8,47} \\
\hline $27,5 \mathrm{~cm}$ & & \multicolumn{2}{|c|}{0,000071543} & \multicolumn{2}{|r|}{$\begin{array}{l}-0,006067 \\
-0,025674\end{array}$} & \multicolumn{3}{|c|}{99,48} & \multicolumn{2}{|l|}{7,27} \\
\hline $32,5 \mathrm{~cm}$ & & \multicolumn{2}{|c|}{0,000037185} & \multicolumn{2}{|r|}{0,000217} & \multirow{2}{*}{\multicolumn{3}{|c|}{$\begin{array}{l}99,06 \\
99,58\end{array}$}} & \multicolumn{2}{|l|}{9,76} \\
\hline $37,5 \mathrm{~cm}$ & & 0,00 & 022664 & \multicolumn{2}{|r|}{0,019259} & & & & 6,51 & \\
\hline $42,5 \mathrm{~cm}$ & & 0,00 & 0030231 & & 0,013167 & & 99,59 & & 6,49 & \\
\hline $47,5 \mathrm{~cm}$ & & 0,00 & 0031119 & & 0,011535 & & 99,65 & & 5,99 & \\
\hline $52,5 \mathrm{~cm}$ & & 0,00 & 0057244 & & $-0,062014$ & & 99,57 & & 6,63 & \\
\hline Total & & 0,00 & 0035156 & & 0,002512 & & 99,51 & & 7,79 & \\
\hline
\end{tabular}

\section{Acuracidade dos modelos não-segmentados na estimativa dos diâmetros}

A Tabela 3 apresenta as estatísticas obtidas pelos modelos para o ajuste total dos dados, desconsiderando as classes diamétricas, em cada posição considerada ao longo do fuste. Os valores calculados das estatísticas para o ajuste que desconsiderou o controle das classes diamétricas foram quase sempre maiores que os valores obtidos para as mesmas estatísticas (não-apresentadas) e posições relativas de medição observadas nas classes com diâmetro maior ou igual a $40 \mathrm{~cm}$. Em contrapartida, os valores das estatísticas calculadas para o ajuste geral foram normalmente inferiores àquelas calculadas para cada uma das cinco classes diamétricas inferiores. Essa observação, embora seja totalmente subjetiva, pode sugerir que um ajuste geral funcione bem para representar os perfis dos fustes das árvores das menores classes diamétricas cujo DAP não ultrapasse $40 \mathrm{~cm}$.

Nas classes com valor central de 37,5, e 42,5, e para o ajuste total, a equação obtida pelo modelo de Goulding e Murray (1976), pelo procedimento "stepwise" sem forçar a entrada de variáveis, propiciou estimativas acuradas dos diâmetros, juntamente com o Polinômio de Potências Fracionárias e Inteiras. No entanto, quando foi forçada a inclusão de variáveis no modelo, a estimativa dos diâmetros na ponta da árvore (partindo de $95 \%$ da altura total) não foi mais possível, pois resultou em uma indeterminação. Como dentre os expoentes cuja entrada estava sendo for çada (expoentes 2, 3, 4 e 5), os expoentes 2, 3 e 4 já eram significativos, apenas o expoente 5 teve, de fato, sua entrada forçada, sendo, pois, o responsável pela indeterminação. Assim, ele foi removido da equação final. Em contrapartida, na classe com valor central 
47,5, os expoentes 2, 3 e 4 também foram significativos pelo procedimento "stepwise" e, nesse caso, foi possível incluir o expoente 5 no modelo. Ao contrário do que aconteceu nas classes de 37,5, 42,5 e para o ajuste total, a inclusão do expoente 5 contribuiu para melhorar a acuracidade da equação que estimou os diâmetros para qualquer altura da árvore.

Com base nas estatísticas mostradas na Tabela 3, foi elaborada a Tabela 4 com as notas atribuídas a cada modelo e o ranking em que o modelo que apresentava o menor valor no somatório das notas atribuídas, era considerado o mais acurado para estimar o diâmetro naquela posição do fuste. Exemplificando: o modelo

TABELA 3: Estatísticas dos modelos polinomiais não-segmentados para estimativa dos diâmetros ao longo do fuste das árvores de Pinus taeda, para o ajuste do conjunto total dos dados, sendo $\mathrm{H}=$ Altura Relativa de Medição, em \%; RP = Resíduo Percentual; Desv = Desvio; SSRR = Soma de Quadrados do Resíduo Relativo e SD = Desvio Padrão das Diferenças.

\begin{tabular}{|c|c|c|c|c|c|c|c|c|c|c|c|c|}
\hline \multirow{3}{*}{$\mathrm{H}$} & \multicolumn{12}{|c|}{ Modelos } \\
\hline & \multicolumn{4}{|c|}{ Hradetzky (1976) } & \multicolumn{4}{|c|}{$5^{0} \mathrm{Grau}$} & \multicolumn{4}{|c|}{ Goulding \& Murray (1976) } \\
\hline & $\mathrm{RP}$ & DESV & SSRR & SD & $\mathrm{RP}$ & DESV & SSRR & $\mathrm{SD}$ & $\mathrm{RP}$ & DESV & SSRR & SD \\
\hline 0 & $-0,354$ & $-0,432$ & 0,211 & 2,834 & 7,562 & 3,333 & 0,522 & 2,413 & $-0,277$ & $-0,268$ & 0,180 & 2,714 \\
\hline 1 & 0,037 & $-0,062$ & 0,084 & 1,620 & $-0,467$ & $-0,276$ & 0,081 & 1,581 & $-1,396$ & $-0,541$ & 0,165 & 2,200 \\
\hline 2 & $-0,140$ & $-0,107$ & 0,046 & 1,099 & $-2,474$ & $-1,044$ & 0,084 & 1,184 & $-0,110$ & 0,016 & 0,115 & 1,757 \\
\hline 3 & $-0,039$ & $-0,090$ & 0,027 & 0,844 & $-2,628$ & $-1,098$ & 0,070 & 0,987 & 0,413 & 0,192 & 0,100 & 1,571 \\
\hline 4 & 0,096 & $-0,042$ & 0,026 & 0,786 & $-2,113$ & $-0,883$ & 0,054 & 0,896 & 0,181 & 0,084 & 0,090 & 1,442 \\
\hline 5 & $-0,395$ & $-0,253$ & 0,032 & 0,858 & $-1,934$ & $-0,825$ & 0,055 & 0,957 & $-0,850$ & $-0,323$ & 0,076 & 1,278 \\
\hline 10 & $-0,086$ & $-0,115$ & 0,063 & 1,219 & 2,107 & 0,653 & 0,087 & 1,144 & -1.110 & $-0,379$ & 0,059 & 1,007 \\
\hline 15 & 0,056 & $-0,032$ & 0,078 & 1,320 & 4,067 & 1,320 & 0,171 & 1,263 & 0,355 & 0,147 & 0,040 & 0,810 \\
\hline 25 & $-0,044$ & 0,017 & 0,159 & 1,736 & 2,367 & 0,783 & 0,185 & 1,710 & 0,534 & 0,273 & 0,039 & 0,847 \\
\hline 35 & $-0,733$ & $-0,156$ & 0,199 & 1,775 & $-1,762$ & $-0,461$ & 0,219 & 1,791 & $-0,783$ & $-0,109$ & 0,052 & 0,830 \\
\hline 45 & $-1,075$ & $-0,175$ & 0,250 & 1,765 & $-3,319$ & $-0,787$ & 0,320 & 1,783 & $-1,258$ & $-0,165$ & 0,085 & 0,971 \\
\hline 55 & $-0,209$ & $-0,014$ & 0,331 & 1,767 & $-0,475$ & $-0,080$ & 0,335 & 1,775 & $-0,081$ & 0,061 & 0,089 & 0,883 \\
\hline 65 & 0,179 & 0,055 & 0,553 & 2,015 & 3,637 & 0,784 & 0,595 & 1,959 & 0,962 & 0,247 & 0,187 & 1,122 \\
\hline 75 & $-0,324$ & $-0,132$ & 0,862 & 1,971 & 5,920 & 0,894 & 0,967 & 1,826 & 0,681 & 0,050 & 0,498 & 1,510 \\
\hline 85 & $-7,603$ & $-0,801$ & 2,582 & 1,902 & $-2,434$ & $-0,279$ & 2,061 & 1,800 & $-7,513$ & $-0,784$ & 2,003 & 1,646 \\
\hline 95 & $-4,844$ & $-0,129$ & 4,863 & 1,084 & $-5,812$ & $-0,166$ & 5,012 & 1,091 & $-17,174$ & $-0,589$ & 6,940 & 1,110 \\
\hline
\end{tabular}

TABELA 4: Notas atribuídas e ranking para os modelos polinomiais não-segmentados para estimativa dos diâmetros ao longo do fuste das árvores de Pinus taeda, elaborados com base nas estatísticas da Tabela 3.

\begin{tabular}{c|c|ccc|}
\hline \multirow{2}{*}{$\begin{array}{c}\text { Alturas relativas } \\
\text { de medição (\%) }\end{array}$} & \multicolumn{3}{|c|}{ Modelos } & \multirow{2}{*}{$1^{\text {o }}$ Lugar } \\
\cline { 2 - 4 } & Hradetzky (1976) & $5^{\text {0 }}$ Grau & Goulding \& Murray (1976) & \\
\hline 1 & 9 & 10 & 5 & $\mathrm{~g}$ \\
2 & 6 & 6 & 12 & $\mathrm{~S} \mathrm{H}$ \\
3 & 6 & 10 & 8 & $\mathrm{H}$ \\
4 & 4 & 10 & 10 & $\mathrm{H}$ \\
5 & 4 & 10 & 10 & $\mathrm{H}$ \\
10 & 4 & 10 & 10 & $\mathrm{H}$ \\
15 & 7 & 11 & 6 & $\mathrm{G}$ \\
25 & 7 & 11 & 6 & $\mathrm{G}$ \\
35 & 7 & 11 & 6 & $\mathrm{G}$ \\
45 & 7 & 12 & 5 & $\mathrm{G}$ \\
55 & 7 & 12 & 5 & $\mathrm{G}$ \\
65 & 7 & 12 & 5 & $\mathrm{G}$ \\
75 & 7 & 11 & 6 & $\mathrm{G}$ \\
85 & 8 & 11 & 5 & $\mathrm{G}$ \\
95 & 12 & 6 & 6 & $\mathrm{~S} \mathrm{H}$ \\
\hline
\end{tabular}


Em que: H = Modelo de Hradetzky (1976); S = Modelo de Schöepfer (1966); G = Modelo de Goulding \& Murray (1976).

polinomial compatível com o modelo volumétrico de Goulding e Murray (1976) sem inclusão de variáveis forçadas, dentre os modelos analisados para o ajuste total dos dados, apresentou, para a altura relativa de medição de $0 \%$, a primeira colocação para a estatística RP, a primeira para a estatística DESV, a primeira para SSRR e a terceira para SD e recebeu então a nota 5, conforme se observa na Tabela 4. A equação na segunda posição, com nota 9, foi a de Hradetzky (1976). Em último, ficou o Polinômio do Quinto Grau, com nota 10. Dessa maneira, na altura relativa de 0\%, o modelo de Goulding e Murray (1976), sem-entrada de variáveis forçadas, foi o que apresentou estimativa mais acurada do diâmetro do fuste, quando o ajuste foi para todos os dados, desconsiderando as classes diamétricas. Já na altura relativa de $95 \%$, o citado modelo ficou em terceiro lugar, com 12 pontos; o Polinômio do Quinto Grau ficou em segundo, com nota 8; e o Polinômio de Potências Fracionárias e Inteiras ficou na primeira colocação, com 4 pontos.

Assim, nessa altura relativa (95\% da altura total), o Polin ômio de Potências Inteiras e Fracionárias (Hradetzky, 1976) foi o que apresentou estimativa mais acurada do diâmetro, quando o ajuste desconsiderou o controle das classes diamétricas.

A Figura 1 mostra os perfis médios reais e estimados pelos três modelos testados, já que a quarta opção é quando se força a entrada de variáveis no modelo de Goulding e Murray (1976) o que não foi possível para o conjunto total dos dados. Para o ajuste geral, desconsiderando o controle das classes diamétricas, os modelos de Hradetzky (1976) e de Goulding e Murray (1976) foram os mais acurados para estimar os diâmetros. Embora na Tabela 5, o Polinômio de Potências Fracionárias e Inteiras tenha ocupado a primeira posição até $10 \%$ da altura, pode-se observar na Figura 1 que, ao longo do restante do fuste, as estimativas obtidas sugerem uma ínfima superestimativa dos diâmetros. Já o polinômio de Goulding e Murray (1976) apresenta uma ínfima superestimativa dos diâmetros de $0 \%$ a $2 \%$ e de $4 \%$ a $25 \%$ da altura. O Polinômio do Quinto Grau mostrou uma certa acuracidade na estimativa dos diâmetros apenas na porção da árvore entre 55 e $85 \%$ da altura total, apresentando uma ligeira superestimativa dos di âmetros partindo desse ponto, e acentuadas tendenciosidades à superestimativa nas porções entre 1 e $5 \%$; e entre 25 e $55 \%$ da altura total; e à subestimativa na base da árvore (até 1\% da altura) e na seção de $10 \%$ a $25 \%$.
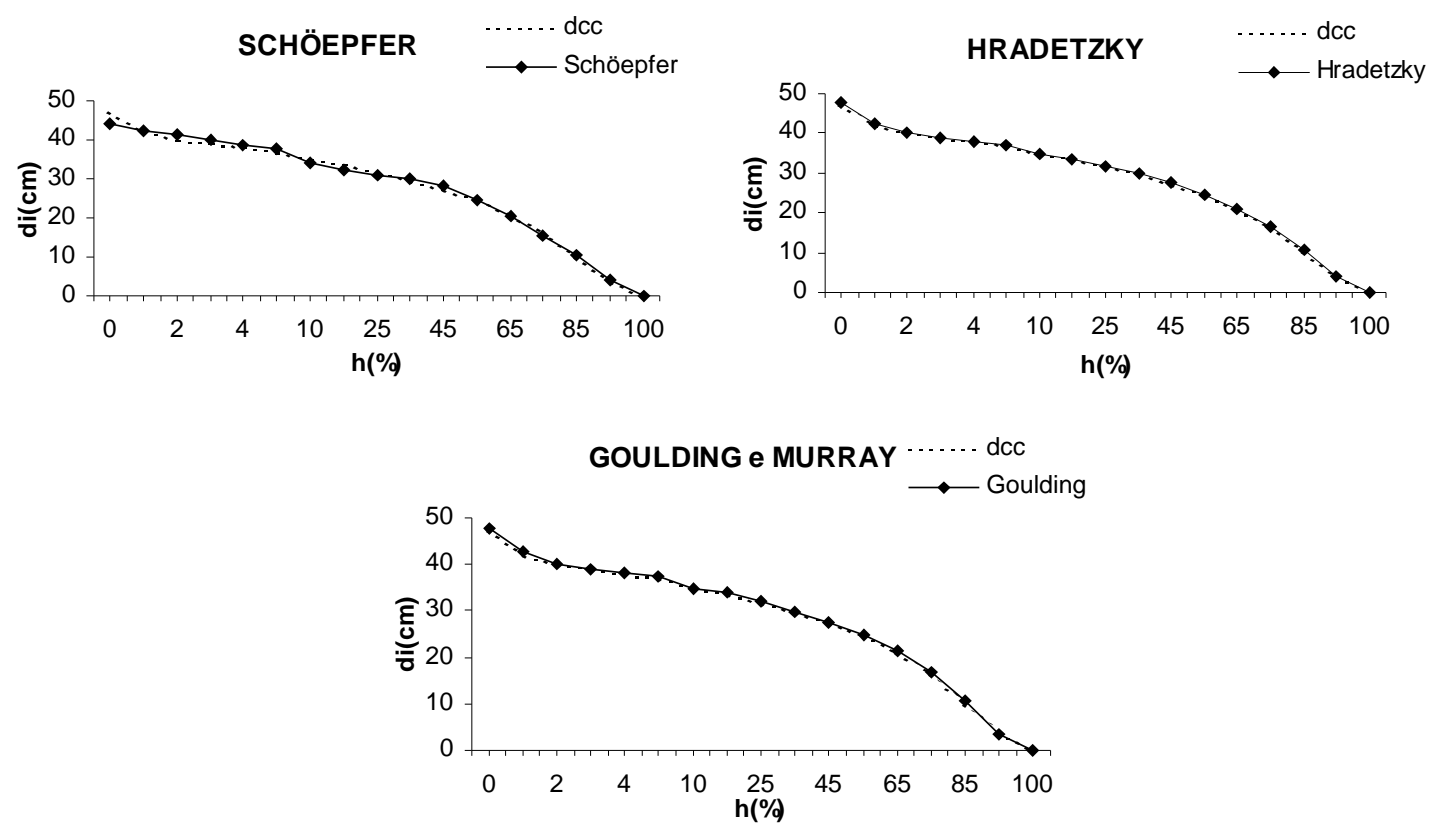

FIGURA 1: Perfis médios para o ajuste desconsiderando as classes diamétricas. 
$\mathrm{Na}$ Tabela 5, encontram-se os resultados do ranking em cada classe diamétrica e em cada posição relativa do fuste. Pôde-se então detectar os modelos mais acurados para estimar os diâmetros em cada uma das posições consideradas.

O Polinômio de Potências Fracionárias e Inteiras destacou-se principalmente na estimativa dos diâmetros da porção inferior das árvores (aproximadamente 10\% da altura total). Esse é um indicativo de que as potências com grandeza de dezena estão modelando bem a base da árvore, concordando com Hradetzky (1976). Uma exceção ocorreu na classe diamétrica de $32,5 \mathrm{~cm}$. Uma outra observação é que o modelo de Goulding e Murray (1976) se destaca sempre na estimativa dos diâmetros partindo de $10 \%$ da altura, seja com ou sem-inclusão de variáveis forçadas no modelo. Considerando que as árvores mais altas utilizadas neste estudo têm cerca de 30 metros de altura, o citado modelo apresenta estimativas menos acuradas do diâmetro apenas para a primeira tora, quando comparado às estimativas propiciadas pelo modelo de Hradetzky (1976).

TABELA 5: Modelo que ocupou o primeiro lugar no ranking em cada posição do fuste por classe diamétrica e para total de árvores.

\begin{tabular}{c|c|c|c|c|c|c|c|c}
\hline \multirow{2}{*}{$\begin{array}{c}\text { Altura } \\
(\%)\end{array}$} & \multicolumn{7}{c}{ Classes Diamétricas } & \multicolumn{7}{c}{. } \\
\cline { 2 - 9 } & 17,5 & 22,5 & 27,5 & 32,5 & 37,5 & 42,5 & 47,5 & 52,5 \\
\hline 0 & $\mathrm{G}$ & $\mathrm{H}$ & $\mathrm{H}$ & $\mathrm{F}$ & $\mathrm{G}$ & $\mathrm{G}$ & $\mathrm{H}$ & $\mathrm{H}$ \\
1 & $\mathrm{H}$ & $\mathrm{H}$ & $\mathrm{H}$ & $\mathrm{S}$ & $\mathrm{H}$ & $\mathrm{H}$ & $\mathrm{H}$ & $\mathrm{S}$ \\
2 & $\mathrm{H}$ & $\mathrm{F}$ & $\mathrm{H}$ & $\mathrm{H}$ & $\mathrm{G}$ & $\mathrm{H}$ & $\mathrm{H}$ & $\mathrm{H}$ \\
3 & $\mathrm{H}$ & $\mathrm{H}$ & $\mathrm{H}$ & $\mathrm{H}$ & $\mathrm{H}$ & $\mathrm{H}$ & $\mathrm{H}$ & $\mathrm{H}$ \\
4 & $\mathrm{H}$ & $\mathrm{H}$ & $\mathrm{H}$ & $\mathrm{H} / \mathrm{F}$ & $\mathrm{H}$ & $\mathrm{H}$ & $\mathrm{H}$ & $\mathrm{H}$ \\
5 & $\mathrm{H}$ & $\mathrm{H}$ & $\mathrm{H}$ & $\mathrm{H}$ & $\mathrm{H}$ & $\mathrm{H}$ & $\mathrm{H} / \mathrm{G}$ & $\mathrm{G}$ \\
10 & $\mathrm{H}$ & $\mathrm{H}$ & $\mathrm{H}$ & $\mathrm{G}$ & $\mathrm{H} / \mathrm{G}$ & $\mathrm{G}$ & $\mathrm{S}$ & $\mathrm{G}$ \\
15 & $\mathrm{H}$ & $\mathrm{H} / \mathrm{G}$ & $\mathrm{F}$ & $\mathrm{H} / \mathrm{F}$ & $\mathrm{G}$ & $\mathrm{G}$ & $\mathrm{G}$ & $\mathrm{F}$ \\
25 & $\mathrm{G}$ & $\mathrm{F}$ & $\mathrm{F}$ & $\mathrm{F}$ & $\mathrm{G}$ & $\mathrm{G}$ & $\mathrm{F}$ & $\mathrm{G}$ \\
35 & $\mathrm{G}$ & $\mathrm{F}$ & $\mathrm{G}$ & $\mathrm{H}$ & $\mathrm{G}$ & $\mathrm{G}$ & $\mathrm{G}$ & $\mathrm{G}$ \\
45 & $\mathrm{G} / \mathrm{F}$ & $\mathrm{F}$ & $\mathrm{F}$ & $\mathrm{F}$ & $\mathrm{G}$ & $\mathrm{G}$ & $\mathrm{F}$ & $\mathrm{F}$ \\
55 & $\mathrm{H} / \mathrm{G}$ & $\mathrm{F}$ & $\mathrm{F}$ & $\mathrm{H} / \mathrm{F}$ & $\mathrm{H} / \mathrm{G}$ & $\mathrm{G}$ & $\mathrm{F}$ & $\mathrm{G}$ \\
65 & $\mathrm{G}$ & $\mathrm{F}$ & $\mathrm{G}$ & $\mathrm{F}$ & $\mathrm{G}$ & $\mathrm{G}$ & $\mathrm{F}$ & $\mathrm{G}$ \\
75 & $\mathrm{G}$ & $\mathrm{H} / \mathrm{F}$ & $\mathrm{F}$ & $\mathrm{F}$ & $\mathrm{G}$ & $\mathrm{G}$ & $\mathrm{F}$ & $\mathrm{F}$ \\
85 & $\mathrm{~F}$ & $\mathrm{~F}$ & $\mathrm{~F}$ & $\mathrm{H} / \mathrm{F}$ & $\mathrm{G}$ & $\mathrm{G}$ & $\mathrm{G}$ & $\mathrm{H}$ \\
95 & $\mathrm{~S}$ & $\mathrm{~S}$ & $\mathrm{H}$ & $\mathrm{F}$ & $\mathrm{H}$ & $\mathrm{H}$ & $\mathrm{H}$ & $\mathrm{F}$ \\
\hline
\end{tabular}

Em que: S = Polinômio do Quinto Grau - Schöepfer (1966); H = Polinômio de Potências Fracionárias e Inteiras Hradetzky (1976); G = Polinômio compatível com modelo volumétrico - Goulding e Murray (1976); F = Polinômio compatível com modelo volumétrico, com entrada de variáveis forçadas.

Para a classe de valor central 17,5 cm, o modelo de Hradetzky (1976) adere quase perfeitamente ao perfil de todo o fuste, apresentando apenas uma ínfima superestimativa dos diâmetros entre $25 \%$ e $65 \%$ da altura total, em que o modelo que aparece em primeiro lugar no ranking é o de Goulding e Murray (1976). Nas demais classes, o comportamento do Polinômio de Potências Fracionárias e Inteiras também é bastante satisfatório, salvo essa tendência à superestimativa dos diâmetros, quase--imperceptível, que aparece na porção média das árvores. Assim, o modelo proposto por Hradetzky (1976) pode ser utilizado com segurança para estimar os diâmetros ao longo de todo o fuste, mesmo nas classes diamétricas e posições relativas em que esse modelo não aparece em primeiro lugar no ranking.

Quanto ao modelo proposto por Goulding e Murray (1976), de um modo geral propiciou estimativas acuradas do diâmetro ao longo do fuste (Figura 2), apesar de apresentar algumas defici ências na estimativa dos diâmetros na ponta da árvore, como aconteceu nas classes com DAP maior ou igual a $45 \mathrm{~cm}$, em que os diâmetros estimados partindo de $95 \%$ da altura total foram indeterminados. Para as classes com di âmetro inferior a $30 \mathrm{~cm}$, o modelo apresentou uma leve superestimativa dos diâmetros na base da árvore (até $20 \%$ da altura total), e uma superestimativa mais acentuada na ponta (acima de $80 \%$ da altura total). Nas classes com diâmetro inferior a $25 \mathrm{~cm}$, a inclusão de variáveis forçadas no modelo melhorou sensivelmente a sua acuracidade na parte superior dos fustes. Já na classe de $27,5 \mathrm{~cm}$, a entrada de variáveis forçadas aparentemente não afetou a acuracidade das estimativas, embora o modelo tenha se destacado no ranking. 
Na classe de 32,5 cm, o Polinômio de Potências Inteiras e Fracionárias e o modelo de Goulding e Murray (1976) apresentaram comportamentos muito semelhantes ao longo de todo o perfil, sendo que a entrada de variáveis forçadas tornou o modelo de Goulding e Murray ainda mais acurado, eliminando uma pequena tendenciosidade à superestimativa existente entre 75 e $85 \%$ da altura total. Assim, as primeiras posições no ranking ficaram divididas entre esses dois modelos, estando ambos aptos para fornecer estimativas confiáveis dos diâmetros ao longo do fuste (Tabela 5). Esse comportamento se repete na classe com valor central de $37,5 \mathrm{~cm}$, sendo que, nesse caso, não houve ajuste do modelo de Goulding e Murray (1976) com entrada de variáveis forçadas, uma vez que tal procedimento gerou estimativas de diâmetros indeterminados partindo de $85 \%$ da altura total o que também aconteceu na classe de $42,5 \mathrm{~cm}$.

Nas duas últimas classes diamétricas, o modelo de Goulding e Murray (1976) só pôde ser analisado com a entrada de variáveis forçadas no modelo, em conseqüência da indeterminação dos diâmetros gerada pelo ajuste normal. Entretanto, a entrada de variáveis forçadas promoveu um excelente ajuste na classe de $47,5 \mathrm{~cm}$, mas provocou uma subestimativa bastante acentuada na base do perfil da classe de $52,5 \mathrm{~cm}$.
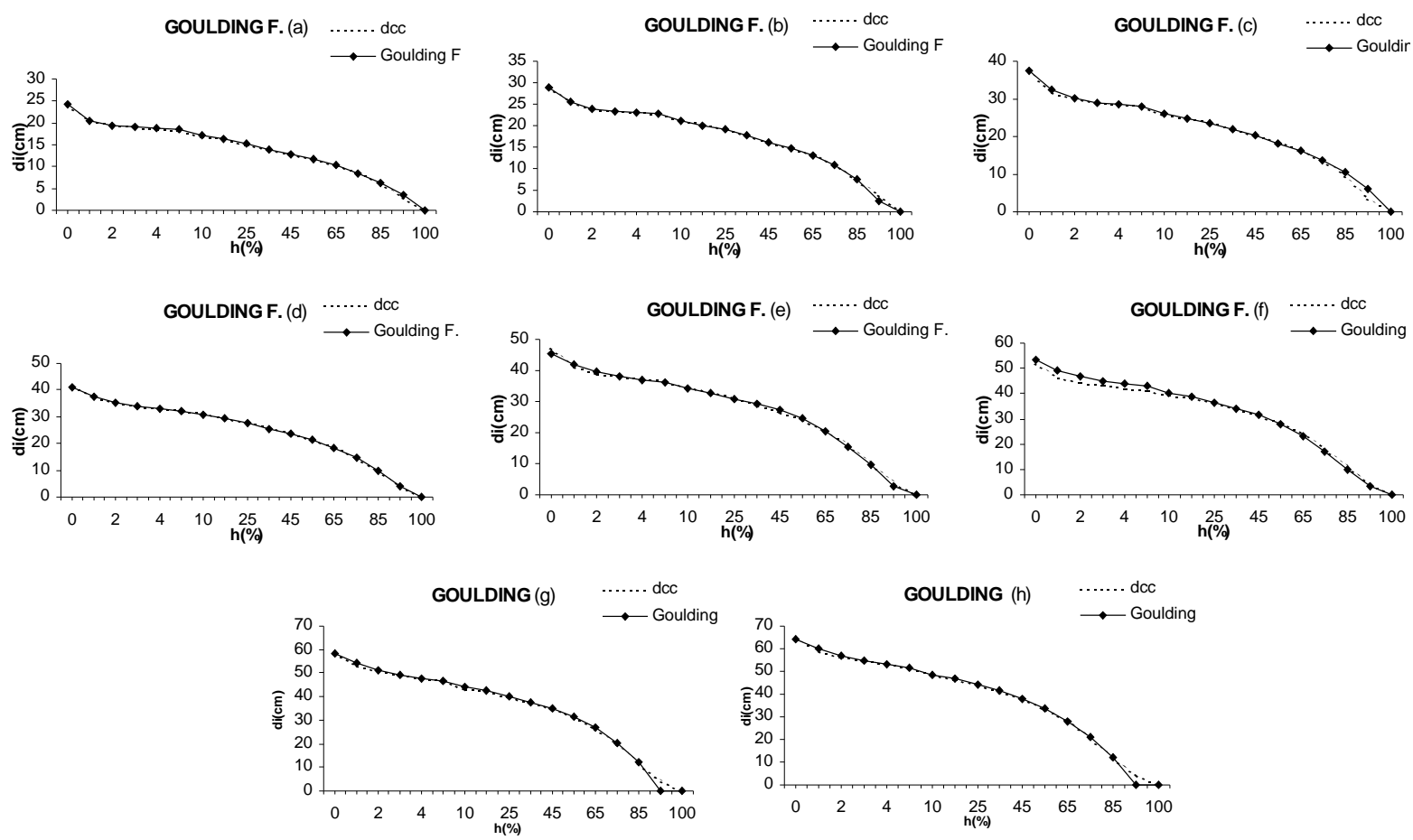

FIGURA 2: Perfis médios para os ajustes por classe diamétrica do modelo de Goulding e Murray (1976), para as classes com valor central de 17,5 cm (a); 22,5 cm (b); 27,5 cm (c); 32,5 cm (d); 37,5 $\mathrm{cm}(\mathrm{e}) ; 42,5 \mathrm{~cm}(\mathrm{f}) ; 47,5 \mathrm{~cm}(\mathrm{~g})$ e $52,5 \mathrm{~cm}(\mathrm{~h})$.

Foi observado que, até a posição de $1 \%$ da altura total, o Polinômio do Quinto Grau (Schöepfer, 1966) subestima acentuadamente os diâmetros, passando, em seguida a superestimá-los até a posição relativa de 5\%. De 10 a 25\% da altura, ocorre uma ligeira subestimativa, comportamento que se repetiu em todas as classes diamétricas. Partindo de $25 \%$ da altura total, o citado modelo assume um comportamento diferenciado em função das dimensões das árvores. Nas classes com diâmetro inferior a $25 \mathrm{~cm}$, estima bem os diâmetros partindo da posição relativa de $25 \%$, embora com acuracidade inferior ao Polinômio de Potências Fracionárias e Inteiras; e tendendo a superestimar os diâmetros na ponta da árvore. Nas classes com diâmetro entre $25 \mathrm{~cm}$ e 44,9 cm, a acuracidade do Polinômio do Quinto Grau diminui ainda mais, ocorrendo ainda uma superestimativa dos diâmetros na porção superior a $85 \%$ da altura total. Para as árvores de maiores dimensões (diâmetro acima de $50 \mathrm{~cm}$ ), o Polinômio do Quinto Grau volta a fornecer boas estimativas diamétricas acima de $25 \%$ da altura total, porém com qualidade inferior à das estimativas 
propiciadas pelo Polinômio de Potências Inteiras e Fracionárias em virtude de ligeiras tendenciosidades à super e subestimativa ao longo do fuste. Observando a Tabela 5, fica clara a inferioridade do Polin ômio do Quinto Grau para estimar os diâmetros ao longo de todo o fuste e em todas as classes diamétricas o que se deve à menor flexibilidade desse modelo em relação aos demais.

\section{Eficiência dos modelos não-segmentados na estimativa dos volumes}

A Tabela 6 mostra as estatísticas obtidas pelos modelos não-segmentados para o ajuste total dos dados, desconsiderando as classes diamétricas em cada posição de medição considerada. Os valores das estatísticas Resíduo percentual (RP), Soma de quadrados do resíduo relativo (SSRR) e Desvio-padrão das diferenças (SD), calculados para as estimativa dos volumes ficaram muito próximos de zero. Já o desvio (DESV) foi pequeno para o modelo de Goulding e Murray (1976) e alto para as demais equações, principalmente para o Polinômio do Quinto Grau.

TABELA 6: Estatísticas dos modelos polinomiais não-segmentados para estimativa dos volumes ao longo do fuste das árvores de Pinus taeda, para o ajuste do total dos dados.

\begin{tabular}{|c|c|c|c|c|c|c|c|c|c|c|c|c|}
\hline \multirow[t]{3}{*}{$\mathrm{H}$} & \multicolumn{12}{|c|}{ Modelos } \\
\hline & \multicolumn{4}{|c|}{ Hradetzky (1976) } & \multicolumn{4}{|c|}{$5^{\circ} \mathrm{Grau}$} & \multicolumn{4}{|c|}{ Goulding e Murray (1976) } \\
\hline & $\mathrm{RP}$ & DESV & SSRR & SD & $\mathrm{RP}$ & DESV & SSRR & SD & $\mathrm{RP}$ & DESV & SSRR & SD \\
\hline 1 & $-0,0030$ & $-3,4855$ & 0,0140 & 0,0049 & 0,0032 & 7,9126 & 0,7545 & 0,0040 & $-0,0002$ & $-0,8800$ & 0,4274 & 0,0042 \\
\hline 2 & $-0,0031$ & $-1,9388$ & 0,0083 & 0,0080 & 0,0017 & 3,2925 & 0,3533 & 0,0065 & $-0,0003$ & $-0,8198$ & 0,3314 & 0,0065 \\
\hline 3 & $-0,0$ & $-1,3$ & 0,0053 & 0 & -0 & 1,0063 & 0,2274 & 0,0083 & 0,0000 & -0, & 39 & 0,0077 \\
\hline 4 & $-0,0035$ & $-1,0598$ & 0,0029 & 0,0088 & $-0,0022$ & $-0,1936$ & 0,1838 & 0,0099 & 0,0000 & $-0,7088$ & 13 & 0,0088 \\
\hline 5 & $-0,00$ & $-1,0^{\prime}$ & 0,0022 & $0,0($ & -0 & $-0,8281$ & 0,1598 & 0,0113 & $-0,0004$ & $-0,9382$ & 0,1940 & 0,0096 \\
\hline 10 & $-0,0093$ & $-1,4236$ & 0,0028 & 0,0160 & $-0,0036$ & 0,1291 & 0,1170 & 0,0169 & $-0,0011$ & $-1,0352$ & 0,1639 & 0,0144 \\
\hline 15 & $-0,0170$ & $-1,9605$ & 0,0050 & 0,0282 & 0,0052 & 2,1371 & 0,1407 & 0,0219 & $-0,0006$ & $-0,9437$ & 0,1736 & 0,0210 \\
\hline 25 & $-0,0$ & & 0,0076 & & 0,0251 & 4,1600 & 0,2388 & & -0 & $-1,3$ & 0,2 & 0,0391 \\
\hline 35 & $-0,03$ & $-2,3$ & 0,0114 & 0,0982 & 0,0 & 3,4989 & 0,2584 & 0,0593 & 0,0006 & 14 & 0,3220 & 0,0600 \\
\hline 45 & $-0,0434$ & $-2,5207$ & 0,0148 & 0,1366 & 0,0213 & 2,0968 & 0,2593 & 0,0788 & 0,0034 & $-1,4267$ & 0,3864 & 0,0798 \\
\hline 55 & $-0,0553$ & $-2,8335$ & 0,0178 & 0,1672 & 0,0168 & 1,4007 & 0,2865 & 0,0953 & 0,0048 & $-1,4931$ & 0,4357 & 0,0966 \\
\hline 65 & $-0,0736$ & $-3,4317$ & 0,0222 & 0,1977 & 0,0218 & 1,6397 & 0,3327 & 0,1098 & 0,0051 & $-1,4941$ & 0,4763 & 0,1108 \\
\hline 75 & $-0,0954$ & $-4,1129$ & 0,0262 & 0,2183 & 0,0292 & 2,1214 & 0,3673 & 0,1204 & 0,0031 & $-1,5513$ & 0,4913 & 0,1211 \\
\hline 85 & $-0,1202$ & $-4,9162$ & 0,0296 & 0,2243 & 0,0327 & 2,5239 & 0,3959 & 0,1241 & 0,0011 & $-1,4278$ & 0,4883 & 0,1239 \\
\hline 95 & $-0,1322$ & $-5,3172$ & 0,0315 & 0,2263 & 0,0327 & 2,6344 & 0,4095 & 0,1249 & 0,0012 & $-1,2546$ & 0,4813 & 0,1242 \\
\hline 100 & $-0,1328$ & $-5,3385$ & 0,0316 & 0,2263 & 0,0317 & 2,5634 & 0,4049 & 0,1258 & 0,0004 & $-1,3256$ & 0,4778 & 0,1251 \\
\hline
\end{tabular}

Em que: $\mathrm{H}=$ Altura Relativa de Medição, em \%; RP = Resíduo Percentual; DESV = Desvio; SSRR = Soma de Quadrados do Resíduo Relativo e SD = Desvio-padrão das Diferenças.

Com base nas estatísticas, foi feita a Tabela $7 \mathrm{com}$ as notas atribuídas a cada modelo e o ranking em que o modelo que apresentou o menor valor no somatório das notas atribuídas foi considerado o mais acurado para estimar o volume naquela posição do fuste. Dessa forma, foi possível determinar qual foi o modelo selecionado para estimar os volumes parciais para todo o conjunto de dados, em cada posi ção relativa do fuste (Tabela 8 ).

Constatou-se que o modelo de Goulding e Murray (1976) apresenta as estimativas mais acuradas dos volumes totais.

A Figura 3 mostra as tendências das estimativas volumétricas para o conjunto total dos dados, evidenciando a superioridade dos modelos de Hradetzky (1976) e Goulding e Murray (1976), em relação ao Polinômio do Quinto Grau. Esse último tende a subestimar os volumes partindo de $85 \%$ da altura total. 
TABELA 7: Notas atribuídas e ranking para os modelos polinomiais não-segmentados para estimativa dos volumes ao longo do fuste das árvores de Pinus taeda, elaborados partindo das estatísticas da Tabela 6.

\begin{tabular}{c|c|c|c|c}
\hline \multirow{2}{*}{$\begin{array}{c}\text { Alturas relativas } \\
\text { de medição (\%) }\end{array}$} & \multicolumn{3}{|c|}{ Modelos } & \multirow{2}{*}{$1^{\circ}$ Lugar } \\
\cline { 2 - 4 } & Hradetzky (1976) & $5^{\circ}$ Grau & Goulding e Murray (1976) & \\
\hline 1 & 8 & 10 & 6 & $\mathrm{G}$ \\
3 & 8 & 9 & 5 & $\mathrm{G}$ \\
4 & 10 & 8 & 6 & $\mathrm{G}$ \\
5 & 8 & 7 & 7 & $\mathrm{~S} \mathrm{G}$ \\
10 & 8 & 8 & 8 & $\mathrm{H} \mathrm{S} \mathrm{G}$ \\
15 & 9 & 8 & 7 & $\mathrm{G}$ \\
25 & 9 & 9 & 6 & $\mathrm{G}$ \\
35 & 9 & 8 & 7 & $\mathrm{G}$ \\
45 & 9 & 8 & 7 & $\mathrm{G}$ \\
55 & 10 & 7 & 7 & $\mathrm{~S} \mathrm{G}$ \\
65 & 10 & 6 & 8 & $\mathrm{~S}$ \\
75 & 10 & 7 & 7 & $\mathrm{~S} \mathrm{G}$ \\
85 & 10 & 7 & 7 & $\mathrm{G}$ \\
95 & 10 & 8 & 6 & $\mathrm{G}$ \\
100 & 10 & 8 & 6 & $\mathrm{G}$ \\
\hline
\end{tabular}

Em que: H = Modelo de Hradetzky (1976); S = Modelo de Schöepfer (1966); G = Modelo de Goulding e Murray (1976).
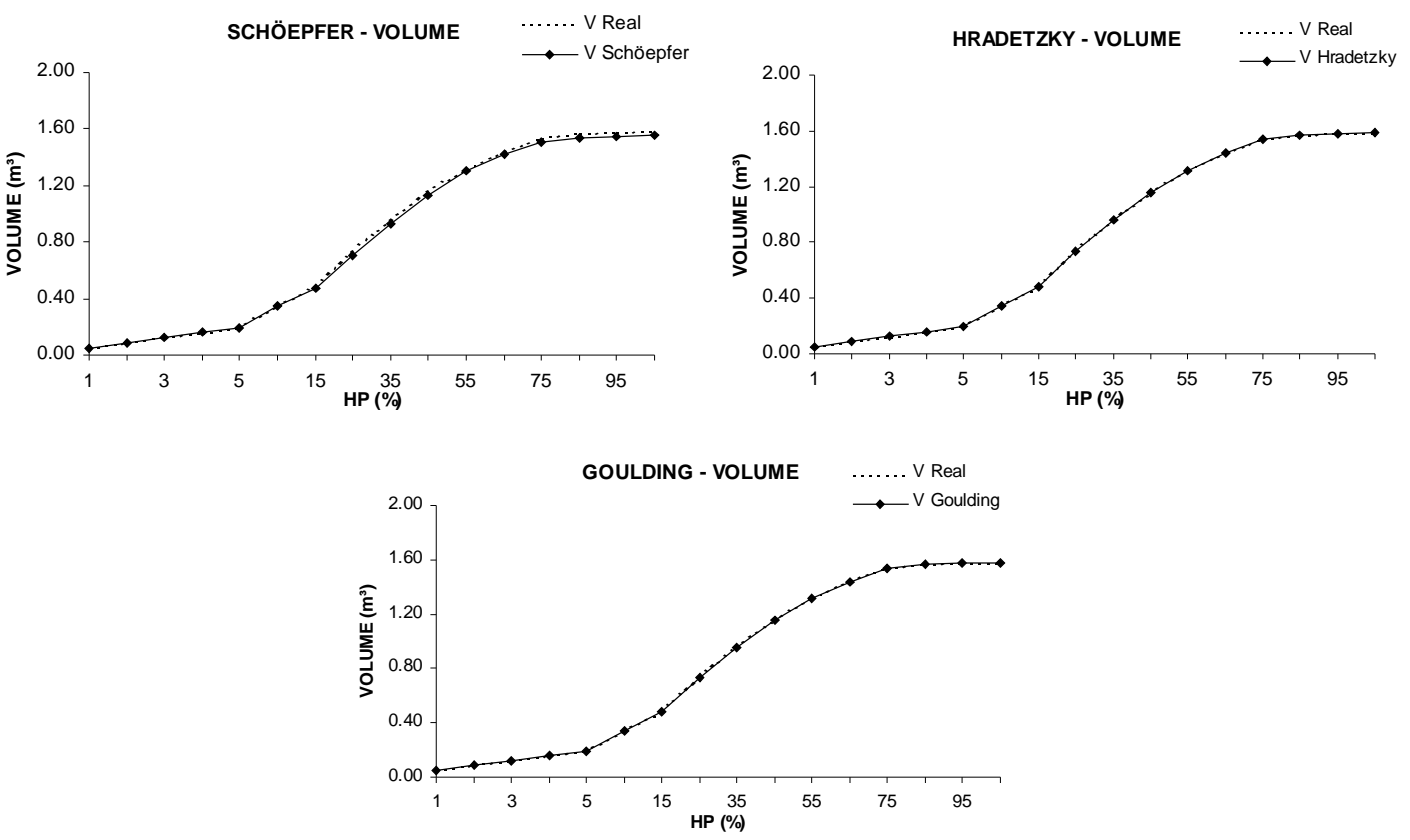

FIGURA 3: Volumes médios reais e estimados pelos modelos polinomiais não-segmentados com ajuste para o conjunto total de dados. 
Na Tabela 8, é apresentado o ranking que permite avaliar qual modelo é mais adequado para estimar os volumes parciais em cada classe diamétrica, em cada posição relativa do fuste. Pode-se observar nessa tabela, que o Polinômio do Quinto Grau (Schöepfer et al., 1966) aparece como mais acurado para estimar os volumes em algumas posições isoladas e para estimar os volumes totais das árvores com diâmetro entre 20 e $30 \mathrm{~cm}$. Entretanto, tal modelo não apresenta nenhuma estabilidade na qualidade das estimativas ao longo de todo o fuste, em nenhuma classe diamétrica o que o torna inflexível e limita o leque de informações confiáveis a serem obtidas. Além disso, quando se trata de estimativa volumétrica, é mais interessante

TABELA 8: Modelo que ocupou o primeiro lugar no ranking para estimativa dos volumes totais e comerciais em cada posição do fuste e em cada classe diamétrica e também para o ajuste desconsiderando o controle das classes diamétricas.

\begin{tabular}{c|c|c|c|c|c|c|c|c}
\hline \multirow{2}{*}{$\begin{array}{c}\text { Altura } \\
(\%)\end{array}$} & \multicolumn{7}{|c}{ Valor Central da Classe Diamétrica } \\
\cline { 2 - 8 } & 17,5 & 22,5 & 27,5 & 32,5 & 37,5 & 42,5 & 47,5 & 52,5 \\
\hline 1 & $\mathrm{G}$ & $\mathrm{G}$ & $\mathrm{H} / \mathrm{G}$ & $\mathrm{F}$ & $\mathrm{G}$ & $\mathrm{G}$ & $\mathrm{F}$ & $\mathrm{H} / \mathrm{G} / \mathrm{F}$ \\
2 & $\mathrm{~S}$ & $\mathrm{~S}$ & $\mathrm{~S} / \mathrm{G}$ & $\mathrm{F}$ & $\mathrm{G}$ & $\mathrm{G}$ & $\mathrm{F}$ & $\mathrm{F}$ \\
3 & $\mathrm{H} / \mathrm{G}$ & $\mathrm{S}$ & $\mathrm{G}$ & $\mathrm{S}$ & $\mathrm{H}$ & $\mathrm{G}$ & $\mathrm{S} / \mathrm{F}$ & $\mathrm{S}$ \\
4 & $\mathrm{H} / \mathrm{G}$ & $\mathrm{H}$ & $\mathrm{H}$ & $\mathrm{F}$ & $\mathrm{H}$ & $\mathrm{G}$ & $\mathrm{H}$ & $\mathrm{H}$ \\
5 & $\mathrm{H}$ & $\mathrm{H}$ & $\mathrm{H}$ & $\mathrm{F}$ & $\mathrm{H}$ & $\mathrm{H}$ & $\mathrm{F}$ & $\mathrm{H}$ \\
10 & $\mathrm{H}$ & $\mathrm{F}$ & $\mathrm{H}$ & $\mathrm{H}$ & $\mathrm{H}$ & $\mathrm{G}$ & $\mathrm{G}$ & $\mathrm{H}$ \\
15 & $\mathrm{H}$ & $\mathrm{G} / \mathrm{F}$ & $\mathrm{H}$ & $\mathrm{H}$ & $\mathrm{H}$ & $\mathrm{G}$ & $\mathrm{S}$ & $\mathrm{G}$ \\
25 & $\mathrm{H}$ & $\mathrm{F}$ & $\mathrm{H}$ & $\mathrm{F}$ & $\mathrm{H}$ & $\mathrm{G}$ & $\mathrm{F}$ & $\mathrm{F}$ \\
35 & $\mathrm{H}$ & $\mathrm{F}$ & $\mathrm{G}$ & $\mathrm{H} / \mathrm{F}$ & $\mathrm{H}$ & $\mathrm{G}$ & $\mathrm{F}$ & $\mathrm{F}$ \\
45 & $\mathrm{H}$ & $\mathrm{G}$ & $\mathrm{G}$ & $\mathrm{S}$ & $\mathrm{H}$ & $\mathrm{G}$ & $\mathrm{H} / \mathrm{F}$ & $\mathrm{F}$ \\
55 & $\mathrm{H}$ & $\mathrm{G}$ & $\mathrm{F}$ & $\mathrm{S}$ & $\mathrm{G}$ & $\mathrm{G}$ & $\mathrm{S} / \mathrm{F}$ & $\mathrm{G}$ \\
65 & $\mathrm{G}$ & $\mathrm{G}$ & $\mathrm{F}$ & $\mathrm{S}$ & $\mathrm{G}$ & $\mathrm{G}$ & $\mathrm{H}$ & $\mathrm{G}$ \\
75 & $\mathrm{G}$ & $\mathrm{G}$ & $\mathrm{F}$ & $\mathrm{S} / \mathrm{F}$ & $\mathrm{G}$ & $\mathrm{G}$ & $\mathrm{H} / \mathrm{F}$ & $\mathrm{F}$ \\
85 & $\mathrm{G}$ & $\mathrm{G}$ & $\mathrm{F}$ & $\mathrm{S}$ & $\mathrm{G}$ & $\mathrm{G}$ & $\mathrm{H}$ & $\mathrm{F}$ \\
95 & $\mathrm{G}$ & $\mathrm{S}$ & $\mathrm{G}$ & $\mathrm{S}$ & $\mathrm{G}$ & $\mathrm{G}$ & $\mathrm{F}$ & $\mathrm{F}$ \\
100 & $\mathrm{G}$ & $\mathrm{S}$ & $\mathrm{G} / \mathrm{F}$ & $\mathrm{S}$ & $\mathrm{G}$ & $\mathrm{G}$ & $\mathrm{H} / \mathrm{G} / \mathrm{F}$ & $\mathrm{G} / \mathrm{F}$ \\
\hline
\end{tabular}

Em que: S = Polinômio do Quinto Grau - Schöepfer (1966); H = Polinômio de Potências Fracionárias e Inteiras Hradetzky (1976); G = Polinômio compatível com modelo volumétrico - Goulding e Murray (1976); F = Polinômio compatível com modelo volumétrico, com entrada de variáveis forçadas.

utilizar um modelo que represente bem a base das árvores, tendo em vista que aí se concentra a maior proporção do volume.

Ainda na Tabela 8, nota-se que os modelos de Hradetzky (1976) e de Goulding e Murray (1976), sendo esse último com ou sem a inclusão de variáves forçadas, se alternam como primeira alternativa para estimar os volumes parciais nas diferentes classes diamétricas. Uma exceção ocorre na classe diamétrica de 40 a 44,9 cm em que o modelo de Goulding e Murray (1976), sem-inclusão de variáveis forçadas se destaca ao longo de todo o fuste.

Analisando as tendências das estimativas por classe diamétrica, constatou-se que o Polinômio de Potências Fracionárias e Inteiras se mostrou bastante eficiente para estimar os volumes parciais, mesmo nas classes e posições relativas em que não ocupou o primeiro lugar no ranking. Os desvios observados foram muito pequenos, cabendo observar apenas uma leve tendência a subestimar os volumes entre 15 e $55 \%$ da altura nas classes de $22,5 \mathrm{~cm}$ e de $52,5 \mathrm{~cm}$, e uma leve superestimativa partindo de $35 \%$ da altura na classe de $32,5 \mathrm{~cm}$. O Polinômio do Quinto Grau (Schöepfer, 1966) só apresentou uma certa acuracidade para estimar os volumes parciais partindo de $55 \%$ da altura total e para as árvores com diâmetro superior a 20 $\mathrm{cm}$. Nas árvores menores, a citada equação subestima sistematicamente os volumes partindo de $5 \%$ da altura.

A entrada de variáveis forçadas no Polinômio de Goulding e Murray (1976), promove um excelente desempenho das estimativas volumétricas propiciadas ao longo de todo o fuste (Figura 4), credenciando -o para ser utilizado nas estimativas dos volumes totais e parciais de todas as classes diam étricas. Deve-se, no entanto, atentar para o fato de que na classe de $32,5 \mathrm{~cm}$, as estimativas são ligeiramente menos acuradas que 
as do Polinômio de Potências Fracionárias e Inteiras e as do mesmo modelo sem a inclusão de variáveis forçadas na porção média dos fustes (entre 25 e $55 \%$ da altura total).

De um modo geral, a inclusão de variáveis forçadas melhora as estimativas volumétricas do modelo de Goulding e Murray (1976), sendo a única exceção na classe de $32,5 \mathrm{~cm}$, em que o ajuste com inclusão de variáveis leva à subestimativa dos volumes a partir de $15 \%$ da altura total.
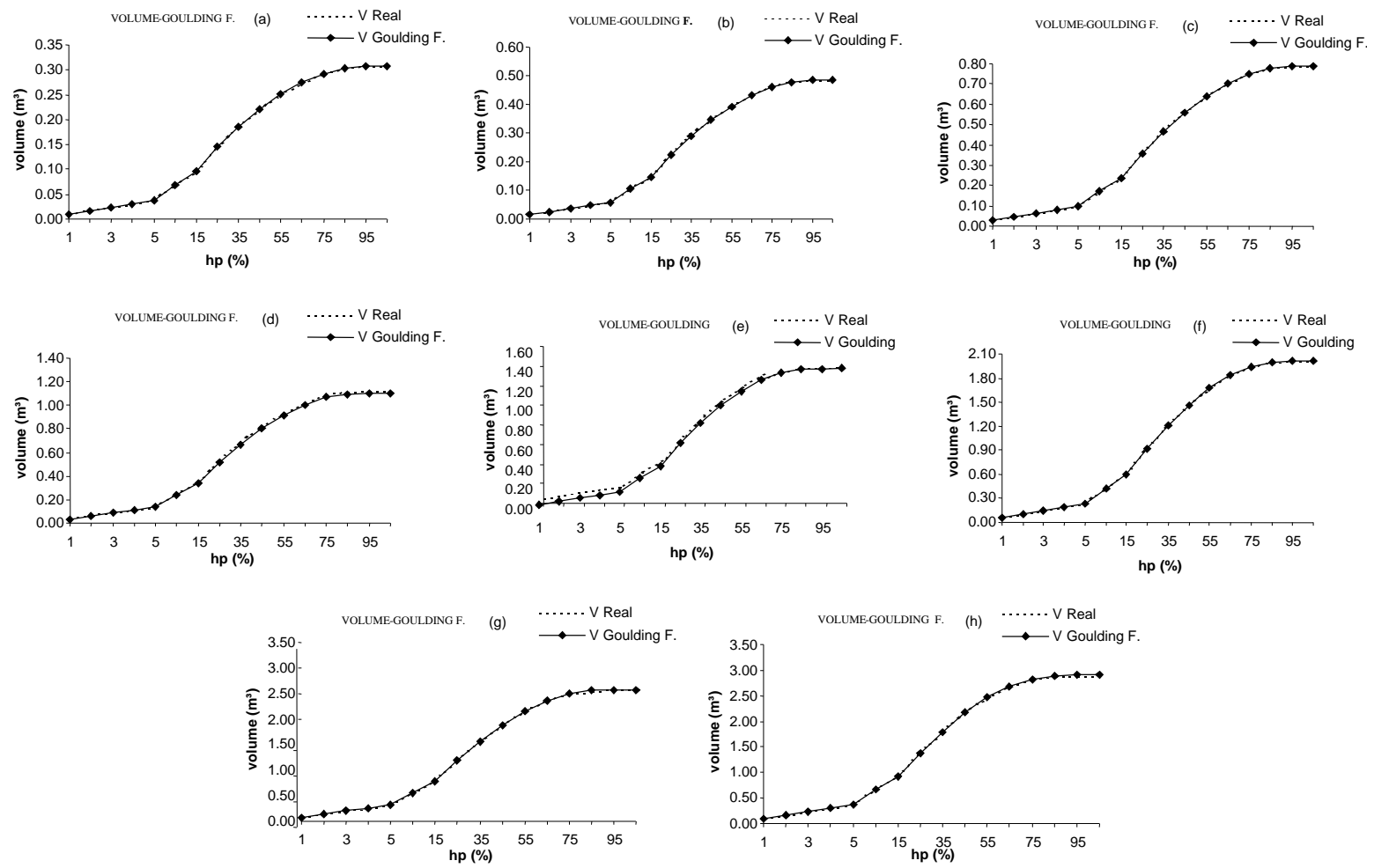

FIGURA 4: Volumes médios reais e estimados para os ajustes por classe diamétrica do modelo de Goulding e Murray (1976), para as classes diamétricas com valor central de 17,5 cm (a); 22,5 cm (b); $27,5 \mathrm{~cm}$ (c); 32,5 cm (d); 37,5 cm (e); 42,5 cm (f); 47,5 cm (g) e 52,5 cm (h).

\section{CONCLUSÕES}

A equação de Potências Fracionárias e Inteiras é a mais estável para estimar os diâmetros ao longo de todo o fuste, enquanto o Polinômio Compatível com o Modelo Volumétrico é o que apresenta estimativas mais acuradas, à exceção da ponta das árvores e em algumas situações da base, em até $2 \%$ da altura total.

Para estimativa dos volumes totais e parciais, por classe diamétrica, o Polinômio Compatível com o Modelo Volumétrico é o que apresenta estimativas mais acuradas, à exceção de alguns casos na ponta das árvores (altura relativa superior a 85\% da altura total). Já o Polinômio de Potências Fracionárias e Inteiras apresenta também estimativas acuradas desses volumes, embora com uma quase impercept ível tendência de subestimá-los.

O Polinômio do Quinto Grau apresentou baixa acuracidade nas estimativas dos di âmetros e dos volumes ao longo do fuste de Pinus taeda na região de estudo, quando comparado com os modelos de Goulding e Murray (1976) e Hradetzky (1976). Não deve, portanto, ser utilizado para nenhuma classe diamétrica nem para o conjunto total dos dados.

Quando o ajuste foi para o total dos dados, sem considerar as classes diamétricas, o Modelo Polinomial Compatível com o Modelo Volumétrico (Goulding e Murray, 1976) e o Modelo de Potências Fracionárias e Inteiras (Hradetzky, 1976) apresentaram estimativas muito acuradas, seja dos di âmetros ao longo do fuste, seja dos volumes totais ou parciais. 


\section{REFERÊNCIAS BIBLIOGRÁFICAS}

AHRENS, S.; HOLBERT, D. Uma função para forma de tronco e volume de Pinus taeda L. Curitiba: EMBRAPA, 1981. p.37-68. (Boletim de Pesquisa Florestal, 3).

ASSIS, A. L. de. Acuracidade na estimativa de volumes comerciais de Eucalyptus grandis e Eucalyptus urophylla. Lavras: UFLA, 1998. 183p. Monografia.

ASSIS, A. L. de "Avaliação de modelos polinomias segmentados e não-segmentados na estimativa de diâmetros e volumes comerciais de Pinus taeda.". 2000. 189p. Dissertação (Mestrado em Engenharia Florestal) - Universidade Federal de Lavras, Lavras.

BORGES, J. F. "Seccionamento do fuste de Pinus taeda L. para obtenção do volume de madeira serrada através da função de forma polinomial”. Floresta, Curitiba, v.13, n.1, p.24-25, 1982.

DEMAERSCHALK, J. P. Integrated systems for the estimation of tree taper and volume. Canadian Journal of Forest Research, Otawa, v.3, n.1, p.90-94, 1976.

FERREIRA, S. O. "Estudo da forma do fuste de Eucalyptus grandis e Eucalyptus cloeziana". 1999. 132p. Dissertação (Mestrado em Engenharia Florestal) - Universidade Federal de Lavras, Lavras.

FIGUEIREDO FILHO, A.; BORDERS, B. E.; HITCH, K. L. Taper equations for Pinus taeda plantations in southern Brazil. Forest Ecology and Management, Amsterdam, v.83, p.36-46, 1996.

FISCHER, F. "Eficiência dos modelos polinomiais e das razões de volume na estimativa volumétrica dos sortimentos e do perfil do fuste de Pinus taeda". 1997. 167p. Dissertação (Mestrado em Engenharia Florestal) - Universidade Federal de Lavras, Lavras.

GOLFARI, L; CASER, R. L; MOURA, V. P. G. "Zoneamento ecológico esquemático para reflorestamento no Brasil": $2^{a}$ aproximação. Belo Horizonte: Centro de Pesquisas Florestais da Regi ão do Cerrado, 1978. 66p. (Série Técnica, 11).

GOULDING, C. J.; MURRAY, J. C. "Polynomial taper equations that are compatible with tree volume equations”. New Zealand Journal of Forest Science, Rotorua, v.5, n.3, p.313-322, Feb. 1976.

HRADETZKY, J. Analyse und interpretation statistisher abr änger keiten. (Biometrische Beitr äge zu aktuellen forschungs projekten). Baden: Württemberg Mitteilungen der FVA, 1976. 146p. (Abt. Biometric und Informatik, 21).

INSTITUTO AGRONÔMICO DO PARANÁ. Cartas climáticas do Estado do Paraná. Londrina, 1994. 49p. (Documentos, 18).

KIRCHNER, F. F.; FIGUEIREDO FILHO, A.; SCOLFORO, J. R. S.; MACHADO, S. A.; MITISHITA, E. A. "O uso de funções spline no cálculo de volumes de árvores." Floresta, Curitiba, v.19, n.1/2, p.116-122, 1989.

KOZAK, A.; MUNRO, D. D.; SMITH, J. H. G. "Taper functions and their aplication in forest inventory. Forest Chronicle, Toronto, v.45, n.4, p.278-283, 1969.

LEITE,H.G.; "Conversão de troncos em multiprodutos da madeira, utilizando programação dinâmica.”. 1994. 230p. Tese (Doutorado em Engenharia Florestal) - Universidade Federal de Viçosa, Viçosa.

LEITE, H. G.; GUIMARÃES, D. P.; CAMPOS, J. C. C. "Descrição e emprego de um modelo para estimar múltiplos volumes de árvores". Revista Árvore, Viçosa, v.19, n.1, p.65-79, 1995.

LIMA, F. S. "Análise de funções de "taper" destinadas à avaliação de multiprodutos de árvores de Pinus elliottii". 1986. 79p. Dissertação (Mestrado em Engenharia Florestal) - Universidade Federal de Viçosa, Viçosa.

McTAGUE, J. P.; BATISTA, J. L. F.; STEINER, L. H. "Equações de volume total, volume comercial e forma do tronco para plantações de Eucalyptus nos Estados de São Paulo e Rio de Janeiro. IPEF, Piracicaba, v.41/42, p.56-63, 1989. 
PARRESOL, B. R.; HOTVEDT, J. E.; CAO, Q. V. "A volume and taper prediction system for bald cypress. Canadian Journal of Forest Research, Otawa, v.17, p.250-259, 1987.

RIOS, M. S. "A eficiência das funções polinomiais, da função spline cúbica, e razões de volume para representar o perfil da árvore e estimar os sortimentos de Pinus elliottii". 1997. 116p. Dissertação (Mestrado em Engenharia Florestal) - Universidade Federal de Lavras, Lavras.

ROSOT, M. A. D. Estudo comparativo de métodos para avaliação volumérica por unidade de área em povoamento de Pinus taeda L. 1989. 163p. Dissertação (Mestrado em Engenharia Florestal) Universidade Federal do Paraná, Curitiba.

SCHÖEPFER,W. Automatisierung des Massen, Sorten und Wertberechnung stenender Waldbestande Schriftenreihe Bad. [S.1.]: Wurtt-Forstl., 1966. n.p.

SCOLFORO, J. R. S.; RIOS, M. S.; OLIVEIRA, A. D. de; MELLO, J. M.; MAESTRI, R. "Acuracidade de equações de afilamento para representar o perfil do fuste de Pinus elliottii". Cerne, Lavras, v.4 , n.1 , p.100$122,1998$.

SILVA, J. A. Funções de forma dos troncos do Pinus taeda, Pinus excelsa, Abies alba e Pinus silvestre. In: SEMINÁRIO SOBRE ATUALIDADES E PERSPECTIVAS FLORESTAIS: o uso de funções de forma de tronco em estudo de volumetria de espécies florestais, 5., 1981, Curitiba (PR). Anais... Curitiba, 1982. p.2945. (Documentos, 9). 
